\title{
NASA /CR-97- 207395 \\ The Scattering Properties of Natural Terrestrial Snows versus Icy Satellite Surfaces
}

\author{
Deborah Domingue \\ Lunar and Planetary Institute, 3600 Bay Area Boulevard, Houston, Texas 77058 \\ E-mail: domingue@lpi.jsc.nasa.gov \\ Beth Hartman ${ }^{1}$ \\ Smith College, Box 6264, 98 Green Street, Northampton, Massachusetts 01063-0100 \\ and \\ Anne Verbiscer ${ }^{2}$ \\ Center for Radiophysics and Space Research, Cornell University, Ithaca, New York 14853 \\ Received April 15, 1996; revised February 27, 1997
}

\begin{abstract}
A. J. Verbiscer and J. Veverka (1990, Icarus 88, 418-428) demonstrated that the single scattering properties of terrestrial snows are highly forward scattering in contrast to the single scattering properties of icy satellite surfaces, which all have a backscattering component to their single scattering functions as modeled with B. Hapke's equations (1981, J. Geophys. Res. 86, 3039-3054; 1984, Icarus 59, 41-59; 1986, Icarus 67, $264-$ 280). This study demonstrates to first order that the differences between the scattering properties of icy satellite regoliths and their terrestrial counterparts are due to differences in particle structure and textures. We find that the particles of the regoliths of the icy satellites are composed of a complex mixture of grain textures and structures. 1997 Academic Press
\end{abstract}

\section{INTRODUCTION}

The bi-directional reflectance variations of the surfaces of icy satellites have been modeled using Hapke's equations (Hapke 1981, 1984, 1986) by a variety of researchers (Buratti 1984, 1985, 1991, Buratti and Veverka 1983, 1984, Buratti et al. 1988, 1990, Domingue et al. 1991, 1995, Helfenstein 1986, Helfenstein et al. 1988, Hillier et al. 1991, Verbiscer and Veverka 1989, Veverka et al. 1987). Verbiscer and Veverka (1990) used this model to fit terrestrial snow reflectance data in a study aimed at comparing the

\footnotetext{
${ }^{1}$ Current address: Department of Earth Sciences, Boston University, Boston, MA 02215

${ }^{2}$ Current address: Department of Physics and Astronomy, University of Massachusetts, Amherst, MA 01003.
}

scattering behavior of terrestrial snows and frost to the scattering behavior of water ice in the outer Solar System. Their results showed that while terrestrial snows and water frost are forward scattering, the surface of the icy satellites are measured to be backscattering. Verbiscer and Veverka (1990) postulated that the source for this difference might be more intricate grain textures caused by the low temperatures and low gravity conditions characteristic of these satellite surfaces. Verbiscer $e$ al. (1990) calculated single particle scattering functions for aerial and intimate mixtures of forward scattering ice and backscattering Cereslike material. Their goal was to study the possibility that the scattering nature of the icy satellites is dominated by this backscattering second component which hides the forward scattering nature of the water ice surface component. Verbiscer et al. (1990) found that the ice itself must be backscattering to produce the results obtained with Hapke's model.

A source of criticism and debate over Hapke's model is its tendency to return backscattering asymmetry parameters when applied to planetary observations and laboratory sample measurements (Mishchenko 1994). However, it should be noted here that when Verbiscer and Veverka (1990) applied this model to terrestrial snow observations it returned forward scattering particle scattering functions.

The source of the scattering difference between terrestrial snows and regolith particles of the icy satellites is not well understood. The laboratory work of McGuire and Hapke (1995) supports the Verbiscer and Veverka (1990) initial hypothesis regarding complicated grain textures. McGuire and Hapke (1995) studied how shape and the 
presence of internal scatterers affect the scattering function of individual particles. The purpose of their study was to examine how a particle that is large compared to the wavelength of incident light (a basic assumption in Hapke's model) scatters light dependent on its physical characteristics. McGuire and Hapke (1995) found that their particles with moderate to high densities of internal scatterers were backscattering in nature. However, the sizes of the artificial particles in their study were on the order of $1 \mathrm{~cm}$ in diameter, which is much larger than most regolith grains. The grain sizes of the water ice/frost component on the icy Galilean satellites, for instance, have been hypothesized to vary from micrometers on Europa to millimeters to centimeters on Callisto based on comparisons of their water ice absorption band depths with laboratory measurements of water ice/frost spectra (Clark 1980, Calvin et al. 1995). While caution needs to be used when applying the findings of the McGuire and Hapke (1995) laboratory studies to modeling results of planetary observations, comparisons with their results are still good for first-order determinations.

At present there is no evidence to support that the difference in scattering behavior between terrestrial snows and icy satellite surfaces is due to differences in grain textures. We present a first-order test of the particle structure and texture hypothesis by comparing the scattering functions of terrestrial snows and icy satellite surfaces with the laboratory results of McGuire and Hapke (1995). McGuire and Hapke (1995) determined that at minimum a twoparameter scattering function was needed to model their single particle scattering measurements, and a double Henyey-Greenstein function modeled their results more accurately than a two-parameter Legendre polynomial. Hartman and Domingue (1997) investigated the effects of the choice of single particle scattering function on the Hapke model by determining whether a two-parameter or a three-parameter double Henyey-Greenstein function better modeled the McGuire and Hapke (1995) measurements and whether the model predicts significantly different results based on their findings with the laboratory measurements. They concluded that the three-parameter double Henyey-Greenstein function did not provide a substantial improvement or difference over the two-parameter double Henyey-Greenstein function when used in Hapke's model. However, Hartman and Domingue (1997) did find that the choice of single particle scattering function incorporated into Hapke's model did influence the values of the remaining model parameters when applied to phase curves of the lunar near side. Therefore we have fitted the terrestrial snow data of Middleton and Mungall (1952) using both double Henyey-Greenstein functions in conjunction with Hapke's model.

Based on Hartman and Domingue's lunar phase curve results, Domingue and Verbiscer (1997) re-analyzed the solar phase curves for the icy Galilean satellites using both two- and three-parameter double Henyey-Greenstein functions. Their goal was to test whether the hemispherical dichotomies in surface structure and texture deduced from Hapke's model were real or a product of the single scattering function incorporated into the model. Domingue and Verbiscer (1997) found that while the absolute values of the model parameters were dependent on the choice of scattering function, the trends in the hemispherical dichotomies predicted by this model were independent of the chosen scattering function and consistent with the exogenic processes modifying these satellite surfaces. Since the terrestrial snow data analyzed by Verbiscer and Veverka (1990) were modeled using a single term HenyeyGreenstein function, as were many of the icy satellites to which they compared their results, we have refitted the terrestrial snow data for ease of comparison with the McGuire and Hapke (1995) laboratory results and with the icy Galilean satellite results from Domingue and Verbiscer (1997). In addition we also reanalyzed Rhea's solar phase curve from Domingue et al. (1995) with a three parameter Henyey-Greenstein single particle phase function for further comparisons with icy Galilean satellite and laboratory results. Finally, we compare our results to the Hartman and Domingue (1997) analysis of the McGuire and Hapke (1995) measurements to infer first-order particle structures for both the terrestrial snows and the icy satellite regolith particles.

\section{DATA}

The terrestrial snow data analyzed by Verbiscer and Veverka (1990) were the in situ goinophotometric measurements of natural snow and frost from Middleton and Mungall (1952). These are the same measurements analyzed here. Middleton and Mungall measured the bidirectional reflectance of six types of naturally occurring snows at reflection angles of $-80^{\circ}$ to $+80^{\circ}$ and incidence angles of $0^{\circ}, 30^{\circ}, 45^{\circ}, 60^{\circ}$, and $75^{\circ}$. Housed in their portable goniophotometer were a collimated light source, which could be positioned at $0^{\circ}, 30^{\circ}, 45^{\circ}, 60^{\circ}$, and $75^{\circ}$ from the surface normal, and a photomultiplier tube that rotated on concentric tracks from $-80^{\circ}$ to $+80^{\circ}$. The light source and photomultiplier were offset in order to prevent obstruction of the light source at very small phase angles. As a result of this displacement, a phase angle of zero was impossible to measure. For more details on the apparatus used for these measurements, the reader is referred to Middleton and Mungall (1952). Five of the natural snows were chosen by Verbiscer and Veverka (1990) for their study (and therefore this project). These are: (1) newly fallen snow, (2) surface frost, (3) rain crusted snow, (4) wind blown snow, and (5) settling snow. The sixth type of snow observed by Middleton and Mungall, "glazed rain crusted snow," was 
TABLE I

Satellite Phase Angle Coverage

\begin{tabular}{llcccc}
\hline \multirow{2}{*}{ Satellite } & $\begin{array}{c}\text { Data } \\
\text { source }\end{array}$ & $\begin{array}{c}0.47-\mu \mathrm{m} \\
\text { Lead }\end{array}$ & $\begin{array}{c}0.47-\mu \mathrm{m} \\
\text { Trail }\end{array}$ & $\begin{array}{c}0.55-\mu \mathrm{m} \\
\text { Lead }\end{array}$ & $\begin{array}{c}0.55-\mu \mathrm{m} \\
\text { Trail }\end{array}$ \\
\hline Europa & Telescopic & $0.2-11.3$ & $0.2-11.3$ & $0.2-11.3$ & $0.2-11.3$ \\
& Voyager & $3.8-119.0$ & $3.0-119.0$ & $3.7-104.4$ & $2.9-94.3$ \\
Ganymede & Telescopic & $0.6-11.4$ & $1.4-11.2$ & $0.4-11.4$ & $1.4-11.2$ \\
& Voyager & $4.8-120.3$ & $20.0-124.0$ & $4.9-32.2$ & $20.0-38.7$ \\
Callisto & Telescopic & $0.4-11.1$ & $0.6-11.2$ & $0.4-11.1$ & $0.6-11.2$ \\
& Voyager & $8.3-50.4$ & $13.9-140.8$ & $8.3-51.7$ & $14.0-107.4$ \\
Rhea & Telescopic & $0.3-6.4$ & $0.9-6.2$ & $0.3-6.4$ & $0.9-6.9$ \\
& Voyager & $13.1-135.5$ & $1.8-16.4$ & $13.3-135.6$ & $1.8-16.6$ \\
\hline
\end{tabular}

Note. Lead corresponds to leading hemisphere, and trail corresponds to trailing hemisphere. All angle ranges are in degrees.

not analyzed since it had a large specular component which Hapke's equations do not model well.

The planetary phase curves modeled in this study include both hemispheres of the icy Galilean satellites and Rhea at 0.47 and $0.55 \mu \mathrm{m}$. The Galilean satellite data and modeling results are taken from Domingue and Verbiscer (1997). The Rhea data and two-parameter double HenyeyGreenstein modeling results are taken from Domingue $e t$ al. (1995). The phase curve data for all the icy satellites are a combination of telescopic observations and Voyager image data. Table I lists the phase angle coverage available for each of these satellites. For a detailed description of the data and data processing the reader is referred to the above references.

\section{ANALYTICAL METHODS}

The model used in this project is based on radiative transfer. An integral part of this model, or any photometric model that attempts to describe scattering from a planetary regolith, is the incorporation of a single particle scattering function. The role of the single particle scattering function is to describe how an average particle or grain from the planetary surface preferentially scatters light in one direction or another. This knowledge is an important tool for providing supporting evidence for various geologic processes such as ion bombardment, where irradiation of an ice surface by ions in a corrotating magnetic field can anneal lattice defects and cause the scattering function to become more forward scattering (Sack et al. 1992). For this project we chose two forms of the double HenyeyGreenstein function: a two-parameter double HenyeyGreenstein function (2P-HG) which is given by

$$
\begin{aligned}
P_{2}(\alpha)= & \left(\left(1-c_{2}\right)\left(1-b_{2}^{2}\right) /\left[1+2 b_{2} \cos (\alpha)+b_{2}^{2}\right]^{3 / 2}\right) \\
& +\left(c_{2}\left(1-b_{2}^{2}\right) /\left[1-2 b_{2} \cos (\alpha)+b_{2}^{2}\right]^{3 / 2}\right),
\end{aligned}
$$

where $\alpha$ is the phase angle and $b_{2}$ and $c_{2}$ are the single particle scattering parameters, and a three-parameter double Henyey-Greenstein function (3P-HG) whose form is given by

$$
\begin{aligned}
P_{3}(\alpha)= & \left(\left(1-c_{3}\right)\left(1-b_{3}^{2}\right) /\left[1+2 b_{3} \cos (\alpha)+b_{3}^{2}\right]^{3 / 2}\right) \\
& +\left(c_{3}\left(1-d_{3}^{2}\right) /\left[1-2 d_{3} \cos (\alpha)+d_{3}^{2}\right]^{3 / 2}\right)
\end{aligned}
$$

where $\alpha$ is the phase angle and $b_{3}, c_{3}$, and $d_{3}$ are the single particle scattering parameters. Note that the $3 \mathrm{P}-\mathrm{HG}$ function defaults to the 2P-HG function for cases where $d_{3}=-b_{3}$. The parameter $c$ in both forms of the double HG-function is a measure of the relative amplitudes of the forward and backscattering components of the single scattering function. The parameters $b$ and $d$ are the widths of the forward and backscattering lobes, respectively, and are defined as the mean cosines of the scattering angle. Therefore the 2P-HG function assumes that the widths of the lobes are identical and just the amplitudes vary.

A modified least squares grid search was used to fit Hapke's disk-resolved equations (Hapke 1984, 1986) to the five types of terrestrial snow observations used in the Verbiscer and Veverka (1990) study. The resulting model parameters are listed in Table II with the corresponding rms values. The rms values are defined such that

$$
\mathrm{rms}=\left\{\left[\sum\left(R_{n}-r_{n}\right)^{2}\right] / N\right\}^{1 / 2},
$$

where $N$ is the number of observational data points, $R$ is the calculated reflectance, $r$ is the observed reflectance, and the sum is over the number of points. Figures 1 through 5 plot our theoretical results versus the data sets.

When Verbiscer and Veverka (1990) fit these data sets they set the opposition amplitude parameter, bo, equal to zero since the snow data show no opposition surge. They also set the roughness parameter, $\theta$-bar, to zero since the types of snows studied here had no visible surface roughness. We chose to let $\theta$-bar vary; however, our results show that indeed these snows are very smooth, consistent with the Verbiscer and Veverka (1990) assumptions. However, we also chose to allow both opposition parameters to vary over our grid search, since the measurements of Middleton and Mungall (1952) contain observations at $i=0.0^{\circ}$ and $e<1^{\circ}$. We found a few percentage differences (up to $2 \%$ in some cases) in the rms values obtained when we allowed bo to vary compared to when we set bo equal to zero. In the model analyses where we set bo $=0$ the resulting values of the remaining parameters were similar to those obtained in the model analyses where bo was allowed to vary as demonstrated by comparing Tables II and III. The most significant differences were in the single scattering function parameters for the newly fallen snow and snow frost. These differences predict a slightly less 
TABLE II

Hapke Parameters for Terrestrial Snow

\begin{tabular}{lllllllllll}
\hline & $\begin{array}{c}\text { Newly } \\
\text { fallen }\end{array}$ & $\begin{array}{c}\text { Snow } \\
\text { frost }\end{array}$ & $\begin{array}{c}\text { Rain } \\
\text { crusted }\end{array}$ & $\begin{array}{c}\text { Wind } \\
\text { blown }\end{array}$ & Settling & & $\begin{array}{c}\text { Newly } \\
\text { fallen }\end{array}$ & $\begin{array}{c}\text { Snow } \\
\text { frost }\end{array}$ & $\begin{array}{c}\text { Rain } \\
\text { crusted }\end{array}$ \\
\hline$w$ & 0.995 & 0.992 & 0.986 & 0.990 & 0.992 & $w$ & 0.995 & 0.993 & 0.986 & 0.990 \\
blown \\
bo & 1.0 & 1.0 & 1.0 & 1.0 & 1.0 & bo & 1.0 & 1.0 & 1.0 & 1.0 \\
$h$ & 0.995 & 0.995 & 0.995 & 0.995 & 0.995 & $h$ & 0.995 & 0.995 & 0.995 & 0.995 \\
Settling
\end{tabular}

Note. Error bars: $w= \pm 0.01$, bo $= \pm 0.05, \mathrm{~h}= \pm 0.005, b_{2}=c_{2}=b_{3}=d_{3}=c_{3}= \pm 0.02, \theta$-bar $= \pm 2$.
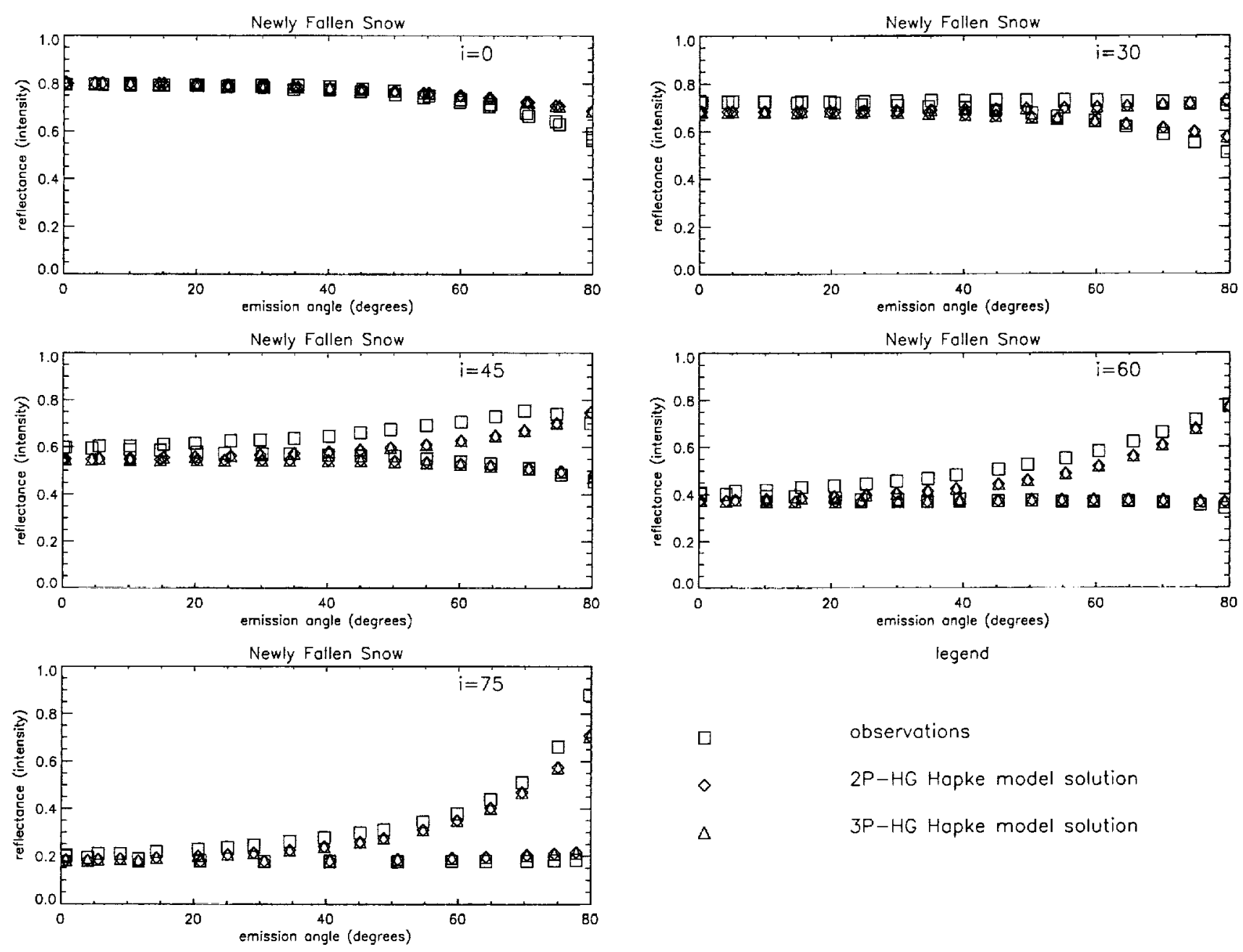

FIG. 1. Comparison of the newly fallen snow reflectance measurements (open squares) of Middleton and Mungall (1952) with the theoretical modeling results using Hapke's model incorporating a two-parameter double Henyey-Greenstein single particle scattering function (open diamonds) and the theoretical modeling results using Hapke's model incorporating a three-parameter double Henyey-Greenstein single particle scattering function (open triangles). 

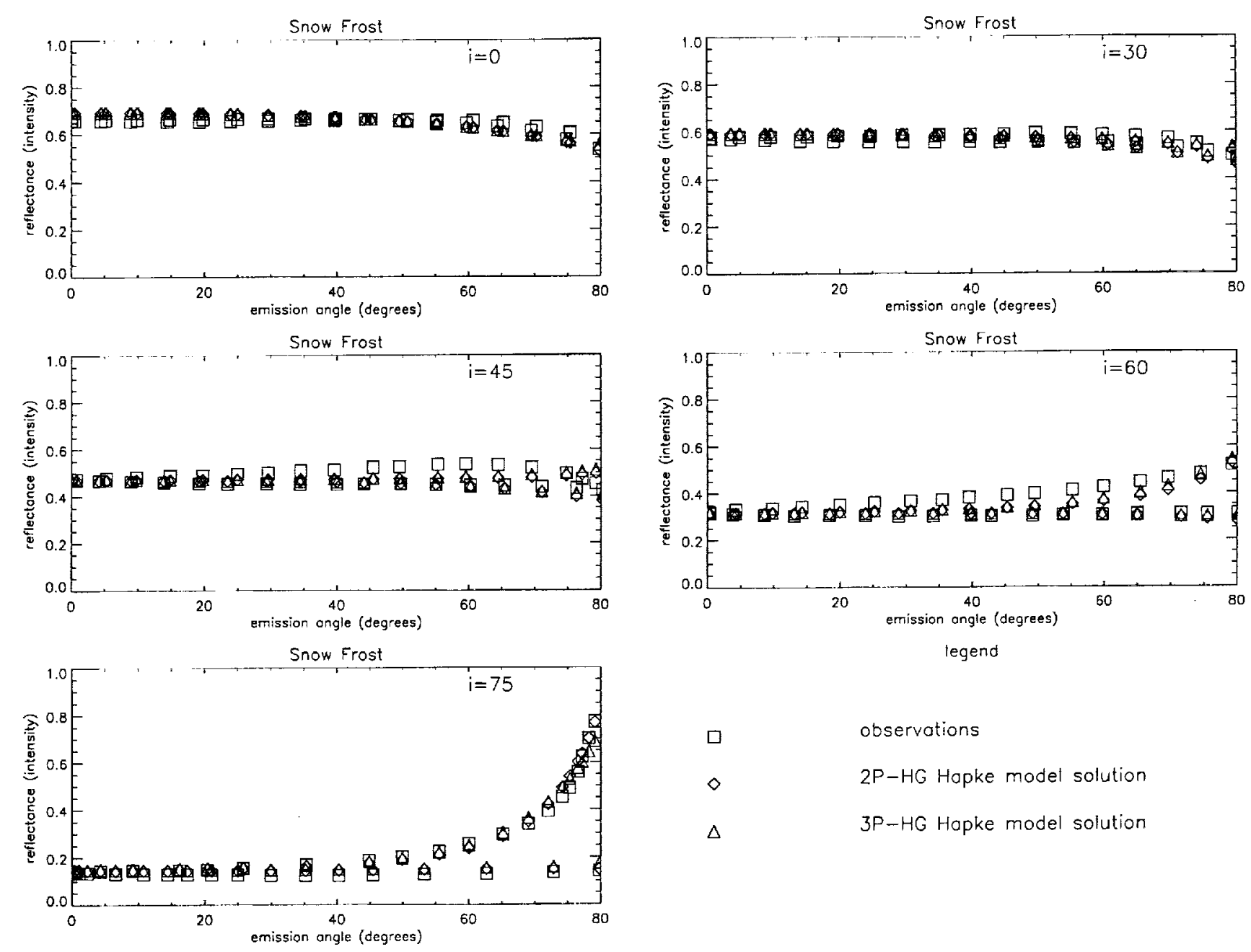

FIG. 2 Same as described in the legend for Fig. 1, for snow frost.

forward scattering function for the newly fallen snow for the bo $=0$ case than for the bo $=1$ case. However, the differences predict a slightly more forward scattering function for the snow frost in the bo $=0$ case than for that in the bo $=1$ case. Our discussions pertain to the bo $=1$ case since there are opposition measurements within the data. The opposition width parameter, $h$, was found to be uniformly wide ( $h=0.995)$ for all the snow data set results where the opposition parameters were allowed to vary. A value of $h \approx 1$ signifies that there is essentially no opposition surge in the snow data.

A modified least-squares grid search was used to fit Hapke's disk-integrated equation (Hapke 1984, 1986) to the disk-integrated phase curves of the three icy Galilean satellites and Rhea. The icy satellite data consist of two phase curves for both leading and trailing hemispheres, one based on observations taken at $0.47 \mu \mathrm{m}$ and the other at $0.55 \mu \mathrm{m}$. Each phase curve was modeled twice, once using Hapke's model with the 2P-HG function and a second time using Hapke's model with the 3P-HG function. The resulting model parameters are listed in Table IV with the corresponding rms values. The rms values are defined such that

$$
\mathrm{rms}=\left\{\left[\sum\left(M_{n}-m_{n}\right)^{2}\right] / N\right\}^{1 / 2}
$$

where $N$ is the number of observational data points, $M_{n}$ is the calculated magnitude from Hapke's theory, $m_{n}$ is the observed magnitude, and the sum is over the number of points. Figures 6 and 7 plot the theoretical models versus observations for the satellite Rhea at 0.47 and $0.55 \mu \mathrm{m}$, respectively. Similar plots for the icy Galilean satellites are shown in Domingue and Verbiscer (1997).

The error bars listed in Table IV are based on the grid 

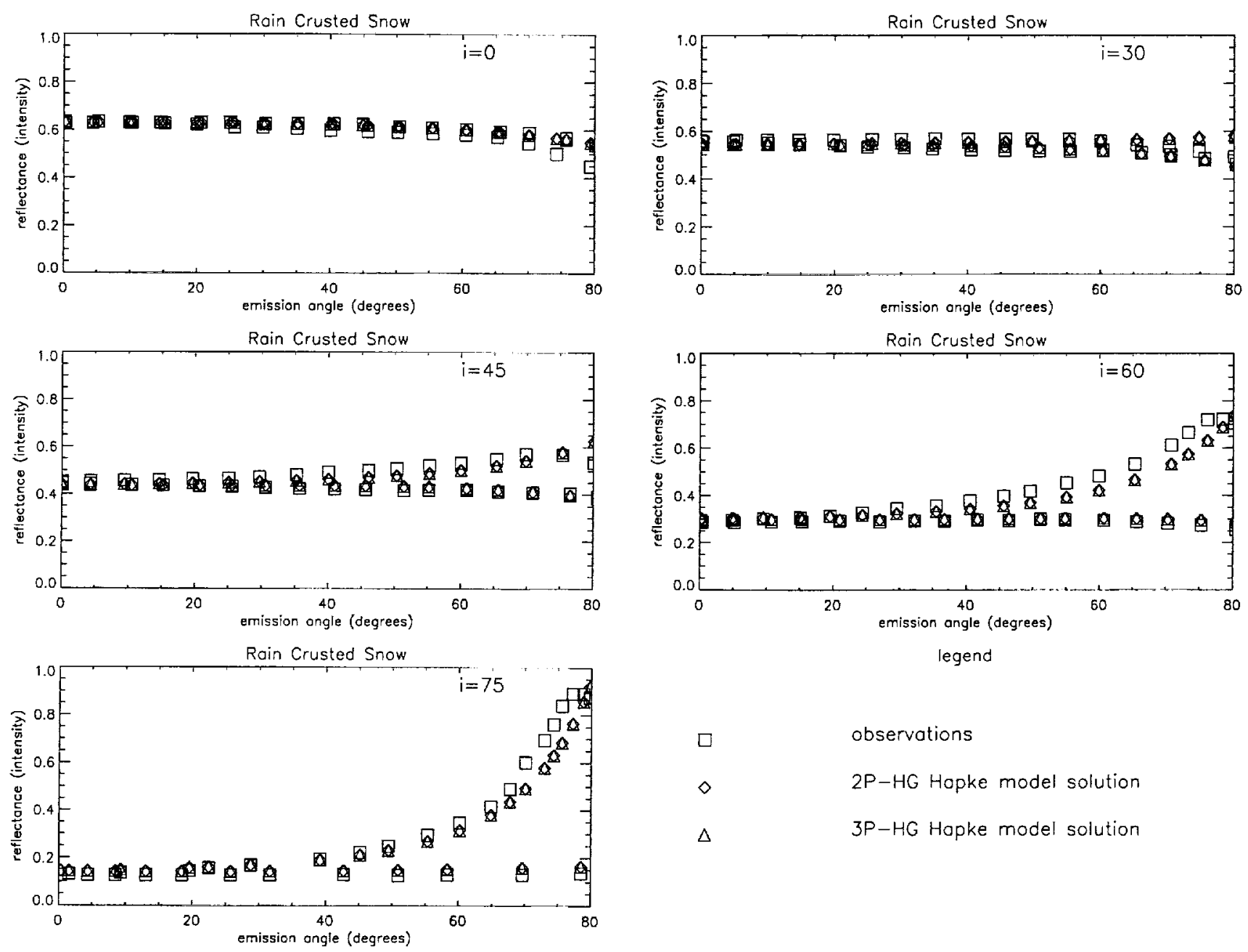

legend

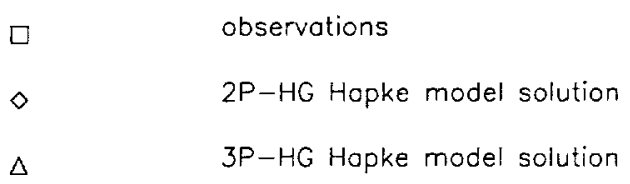

FIG. 3. Same as described in the legend for Fig. 1, for rain crusted snow.

size of the modified least-squares fitting routine. The details of this fitting routine are described in Domingue and Verbiscer (1997). However, the phase angle coverage in the satellite phase curves is also important in establishing the quality of fit for many of the parameters. A detailed error analysis of the fits to the icy Galilean satellites is given in Domingue and Verbiscer (1997) which is also applicable to Rhea. The final error bars for each of the model parameters are given in Table $\mathrm{V}$.

\section{RESULTS}

\section{Terrestrial Snows}

Hapke's equations model the terrestrial snow observations reasonably well, as demonstrated in Figs. 1 through 5. Comparisons of the results between the $2 \mathrm{P}-\mathrm{HG}$ and the 3P-HG models are surprisingly similar. Both models predict high values of the single scattering albedo $(w)$ which are within the error bars $( \pm 0.002)$ of each other. In addition, both models predict very smooth values for the macroscopic roughness parameter, $\theta$-bar. In general, values for $\theta$-bar lie in the range $0-3^{\circ}$, all approximately within the error bars $\left( \pm 2^{\circ}\right)$; however, the 3P-HG model for snow frost predicts a rougher surface than the $2 \mathrm{P}-\mathrm{HG}$ model.

Both models predict the same value for the opposition width parameter, $h$, for all snow types. The high value of $h$ is consistent with the lack of an opposition surge in the snow data. Hapke's model (1986) correlates the value of $h$ to porosity within the optically active layer of the regolith. However, to obtain an accurate estimate of the regolith porosity a reasonable measure of $h$ is needed. Due to the lack of the detection of an opposition surge in the snow data, the value of $h$ obtained in the modeling results cannot be accurately correlated to porosity.

The opposition amplitude parameter, bo, can be correlated to the opacity of the regolith particles. It is a measure 

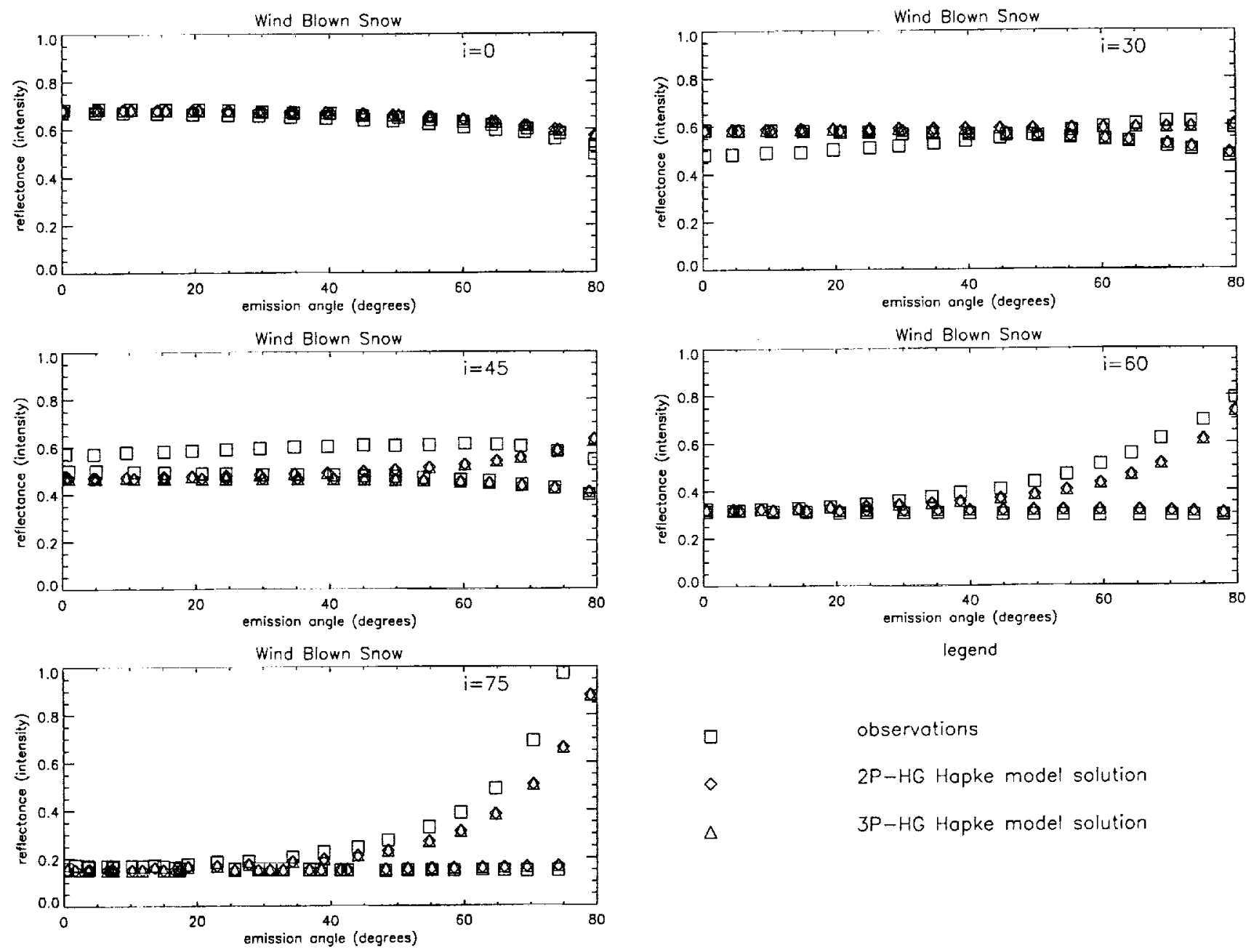

FIG. 4. Same as described in the legend for Fig. 1, for wind blown snow.

of the percentage of incident light which is scattered at the surface; thus, a value of 1 signifies that all the incident light is scattered at the surface and the particle is opaque. The value of bo is known to influence the shape of the disk-integrated phase curve out to $30^{\circ}$ for which the terrestrial snow data sets have corresponding measurements. However, the value of bo is influenced by the value of $h$. Since there was no detection of an opposition surge in the snow observations and $h$ has a large value corresponding to the lack of an opposition surge, no interpretation of the quantitative values of bo can be made.

The single scattering functions from both models are plotted in Fig. 8. They both predict highly forward scattering behavior for terrestrial snow and water frost, consistent with the results of Verbiscer and Veverka (1990), who fit the snow data with a version of Hapke's model which incorporated a single Henyey-Greenstein function. No significant differences are seen in the single particle scattering function predicted by Hapke's model incorporating the 2P-HG function versus this same model incorporating the 3P-HG function. Except for the newly fallen snow data, the values of $c_{2}$ and $c_{3}$ are uniformly zero. When $c_{2}$ and $c_{3}$ are zero, the 2P-HG and $3 \mathrm{P}-\mathrm{HG}$ functions default to the single Henyey-Greenstein function used by Verbiscer and Veverka (1990). Even the $c_{2}$ and $c_{3}$ values for the newly fallen snow are zero within the error bars. Our results also agree with the values for the single scattering function found by Verbiscer and Veverka (1990). We attribute this lack of a backscattering component to the unique scattering nature of snow.

\section{Satellite Surfaces}

Buratti (1991) modeled both hemispheres of Ganymede and Callisto using Hapke's model to look for hemispherical variations in their surface photometric properties. The val- 

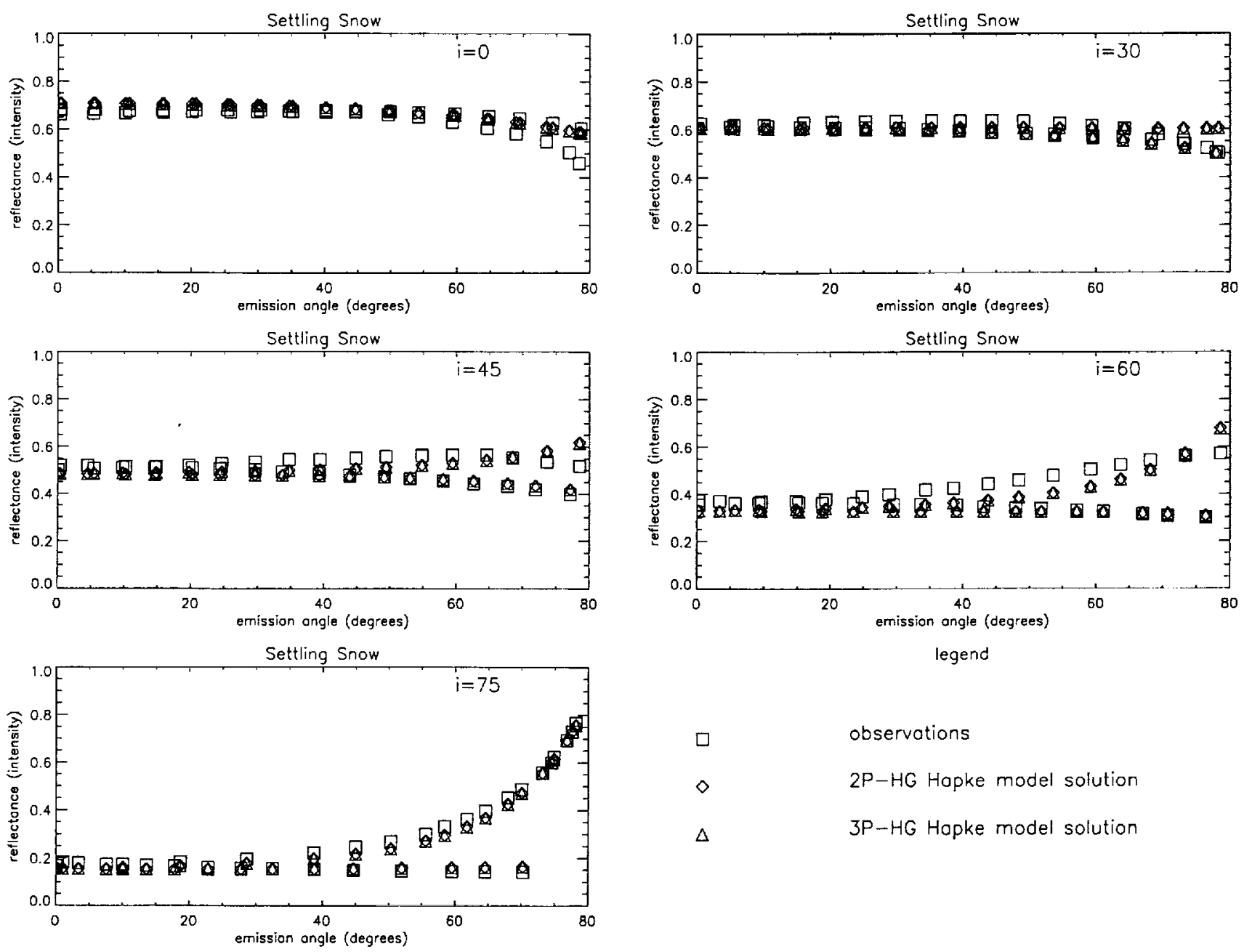

FIG. 5. Same as described in the legend for Fig. 1, for settling snow.

ues quoted by Buratti for the surface roughness parameter are commensurate with our Ganymede results, but greatly differ from our Callisto results. Our values for the single scattering albedo agree with Buratti's values for Ga- nymede's leading and Callisto's trailing hemispheres; however, they disagree with her values for Ganymede's trailing and Callisto's leading hemispheres. We attribute these differences to the different single particle scattering functions

TABLE III

Hapke Parameters for Terrestrial Snow with bo $=0$

\begin{tabular}{lllllllllll}
\hline & $\begin{array}{c}\text { Newly } \\
\text { fallen }\end{array}$ & $\begin{array}{c}\text { Snow } \\
\text { frost }\end{array}$ & $\begin{array}{c}\text { Rain } \\
\text { crusted }\end{array}$ & $\begin{array}{c}\text { Wind } \\
\text { blown }\end{array}$ & Settling & & $\begin{array}{c}\text { Newly } \\
\text { fallen }\end{array}$ & $\begin{array}{c}\text { Snow } \\
\text { frost }\end{array}$ & $\begin{array}{c}\text { Rain } \\
\text { crusted }\end{array}$ & $\begin{array}{c}\text { Wind } \\
\text { blown }\end{array}$ \\
Settling
\end{tabular}

Note. Error bars: $w= \pm 0.01, b_{2}=c_{2}=b_{3}=d_{3}=c_{3}= \pm 0.02, \theta$-bar $= \pm 2$. 
TABLE IV

Hapke Parameters for the Icy Galilean Satellites and Rhea

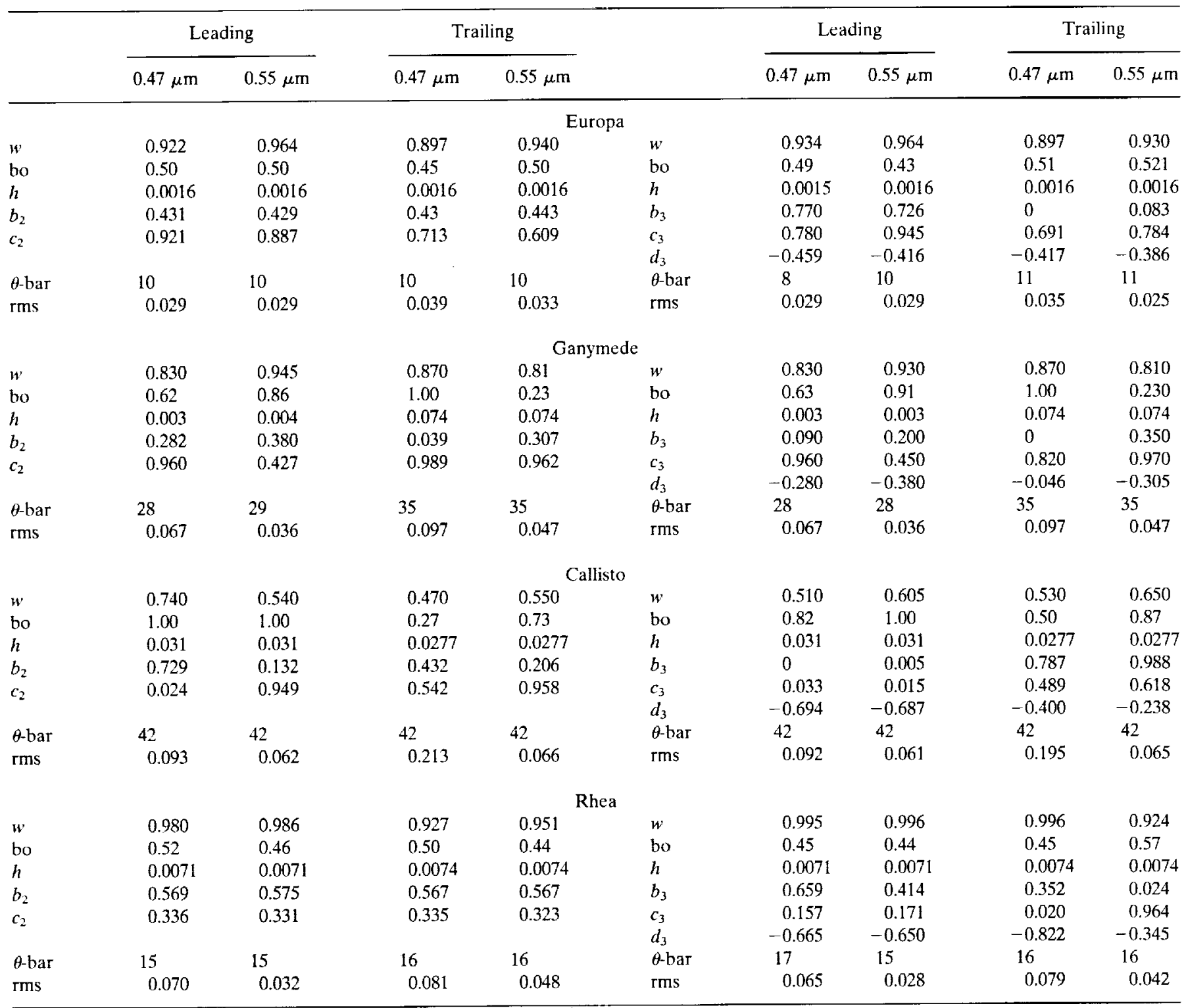

Note. Error hars: $w= \pm 0.01$, bo $= \pm 0.01, h= \pm 0.001, b_{2}$ and $b_{3}= \pm 0.005, c_{2}$ and $c_{3}= \pm 0.005, d_{3}= \pm 0.005, \theta-\mathrm{bar}= \pm 2$.

incorporated into the models. Buratti used a single term Henyey-Greenstein function in her analysis; therefore, her results are not easily compared with those stated in this paper. Helfenstein et al. (1996) modeled Callisto's surface using the same model with a 3P-HG single scattering function. Their results differ from those stated in this paper; however the error bars quoted by Helfenstein et al. (1996) place our results close to theirs. The major difference between their analysis and ours is that they allowed the opposition amplitude parameter, bo, to have values greater than 1 . This will affect the values of the remaining model parameters; therefore in the following discussions we will not compare our results with theirs in order to maintain internal consistency.

The values for the single scattering albedo between the two models are within the error bars for the satellites Europa and Ganymede. The only differences in $w$ seen for Europa were between the $0.47-\mu \mathrm{m}$ leading hemisphere results and the $0.55-\mu \mathrm{m}$ trailing hemisphere results. The only difference in $w$ seen for Ganymede was between 0.55 $\mu \mathrm{m}$ leading hemisphere results. The two models returned significantly different results for both hemispheres of Callisto at both wavelengths of observation; however, the leading hemisphere of this satellite has no large phase angle 

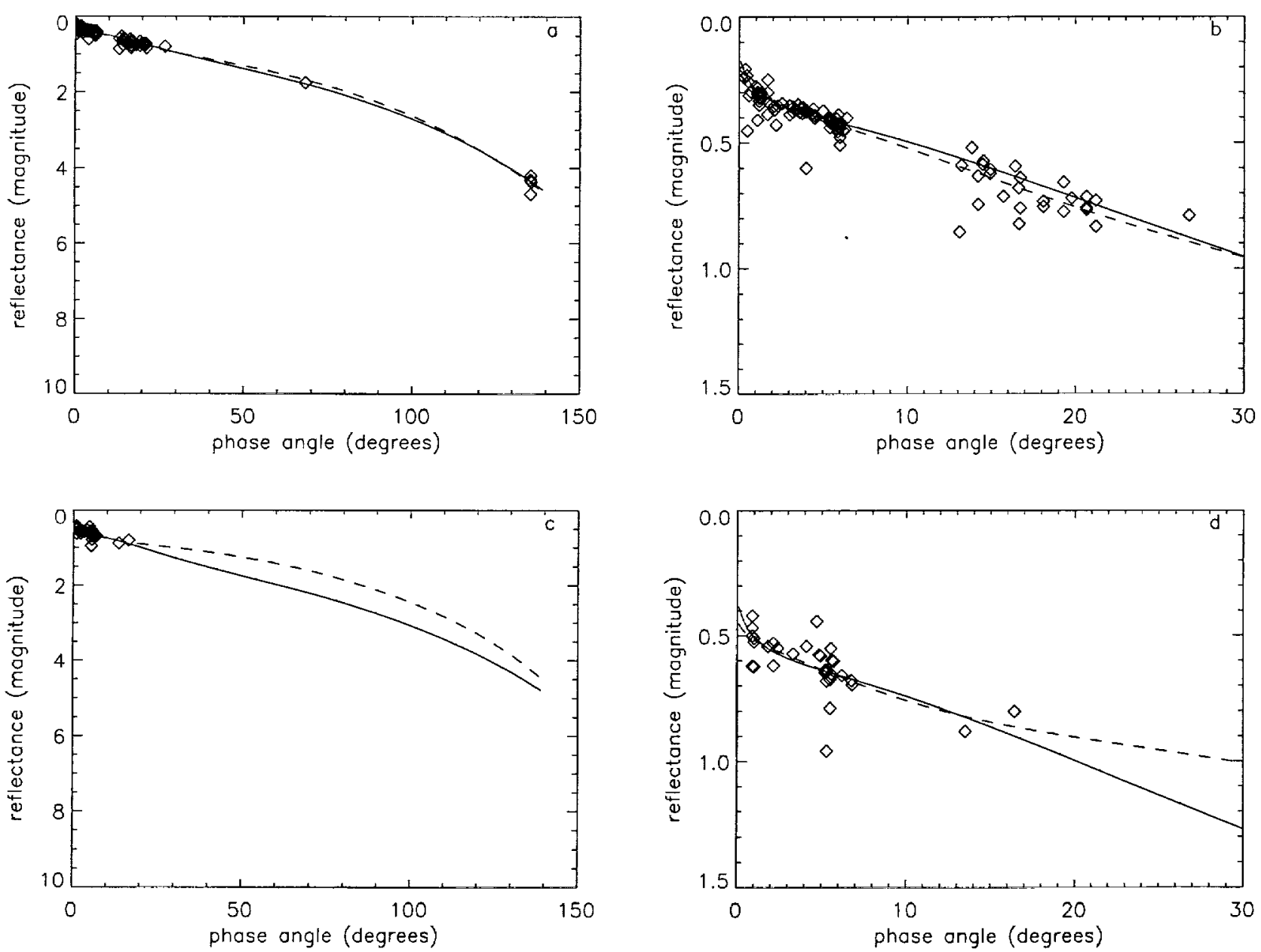

FIG. 6. The solar phase curves of Rhea's leading ( $a$ and b) and trailing ( $c$ and $d$ ) hemispheres at $0.47 \mu \mathrm{m}$, where the diamonds represent the disk-integrated observations, the solid line represents the Hapke model solution incorporating a two-parameter HG function, and the dashed line represents the Hapke model solution incorporating a three-parameter HG function.

data to constrain its phase curve at these important viewing geometries. The large phase angle data constrain the values of $w, \theta$-bar, and the single particle scattering function parameters. There were no consistent trends in the Callisto results. The two models also returned significantly different values of $w$ for Rhea's trailing hemisphere. The leading hemisphere data set values for $w$ were within the error bars; however, there are significant differences in $w$ between the two models for the trailing hemisphere data sets. This is not surprising since like Callisto's leading hemisphere, Rhea's trailing hemisphere has no large phase angle data to constrain the model parameter values.

The value of the surface macroscopic roughness parameter, $\theta$-bar, showed no significant variation with model in the results for the icy satellites. The only other Hapke parameter for which there were no significant variations with model was the opposition width parameter.

The opposition amplitude parameter, however, does show some significant differences between the two models. We define a significant difference in bo to be a difference of 0.1 which is based on variations in rms as bo varies while the other parameters remain constant. Changes in rms of $5 \%$ are considered significant. The only satellites showing a signifcant difference in bo between the two model results were Callisto and Rhea. The significant differences in bo values in the Rhea modeling results were for the trailing hemisphere at $0.55 \mu \mathrm{m}$ only and can be attributed to the scatter in the opposition measurements. However, the opposition data for Callisto have remarkably little scatter; therefore this difference in opposition parameters is not readily understood. These variations in bo with model for these satellites are consistent with the terrestrial snow results.

The single particle scattering functions show strong variations with choice of model in the satellite solutions. Figures 9 through 12 plot the scattering functions for Europa, 

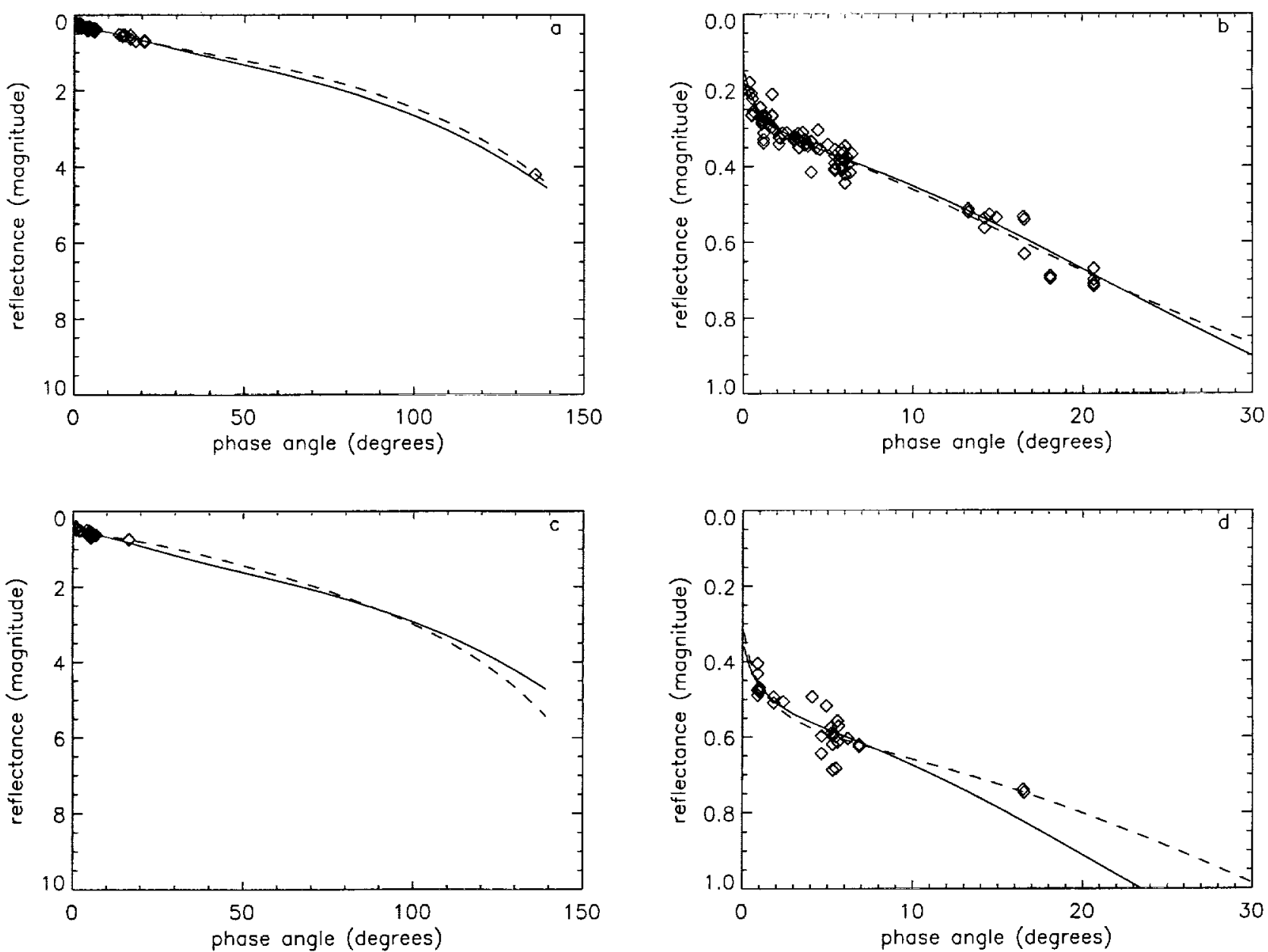

FIG. 7. Same as described in the legend for Fig. 6, at $0.55 \mu \mathrm{m}$.

TABLE V

Error Bars

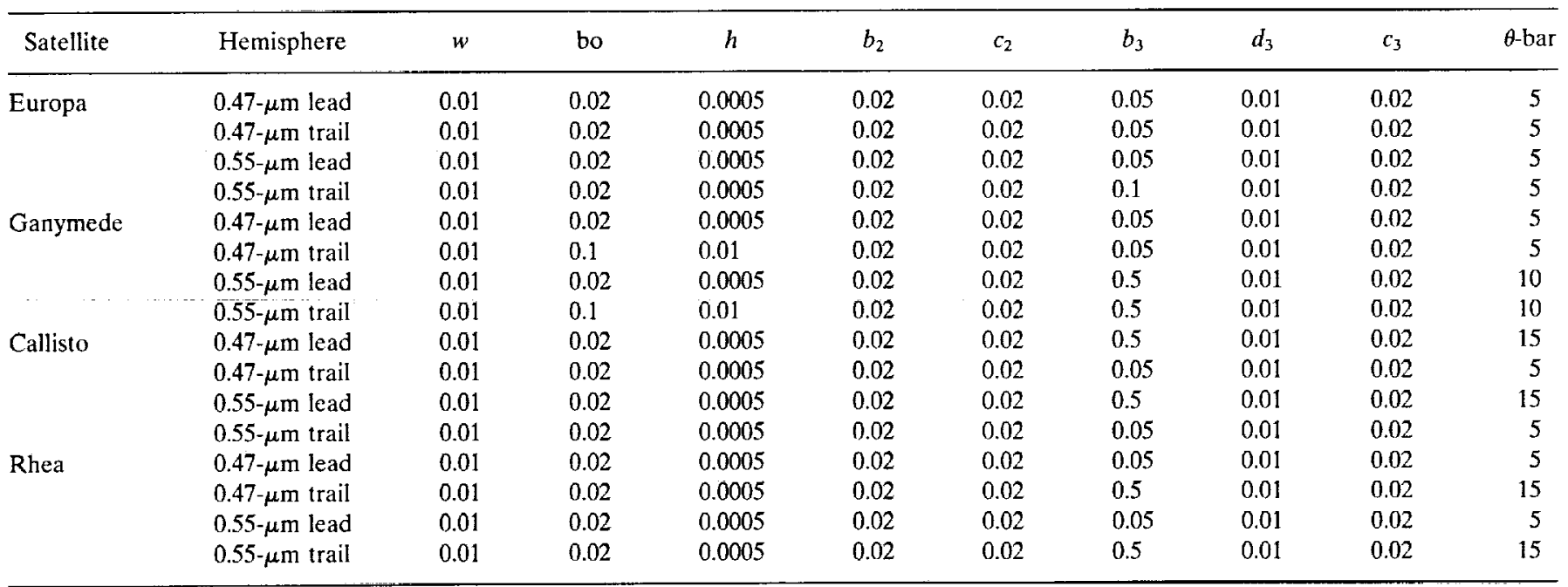


TABLE VI

Isotropic vs Anisotropic Multiple Scattering Model Results for Rhea

\begin{tabular}{|c|c|c|c|c|c|c|c|c|}
\hline \multirow[b]{3}{*}{$w$} & \multicolumn{4}{|c|}{ Leading at $0.47 \mu \mathrm{m}$} & \multicolumn{4}{|c|}{ Leading at $0.55 \mu \mathrm{m}$} \\
\hline & \multicolumn{2}{|c|}{ Isotropic } & \multicolumn{2}{|c|}{ Anisotropic } & \multicolumn{2}{|c|}{ Isotropic } & \multicolumn{2}{|c|}{ Anisotropic } \\
\hline & 0.995 & \pm 0.01 & 0.9909 & \pm 0.0009 & 0.996 & \pm 0.01 & 0.9935 & \pm 0.0002 \\
\hline bo & 0.45 & \pm 0.01 & 0.739 & \pm 0.8337 & 0.44 & \pm 0.01 & 0.549 & \pm 0.2399 \\
\hline$h$ & 0.0071 & \pm 0.001 & 0.0539 & \pm 0.232 & 0.0071 & \pm 0.001 & 0.0041 & \pm 0.0029 \\
\hline$b_{3}$ & 0.659 & \pm 0.005 & 0.4828 & \pm 0.07 & 0.414 & \pm 0.005 & 0.3394 & \pm 0.1263 \\
\hline$c_{3}$ & 0.157 & \pm 0.005 & 0.17 & \pm 0.09 & 0.171 & \pm 0.005 & 0.17 & \pm 0.03 \\
\hline$d_{3}$ & -0.665 & \pm 0.005 & -0.5976 & \pm 0.07 & -0.650 & \pm 0.005 & -0.6492 & \pm 0.0071 \\
\hline$\theta$-bar & 17 & \pm 2 & 19 & \pm 0.7 & 15 & \pm 2 & 15.6 & \pm 0.8 \\
\hline rms & 0.065 & & 0.065 & & 0.028 & & 0.028 & \\
\hline
\end{tabular}

Ganymede, Callisto, and Rhea, respectively. The 3P-HG model predicts a forward scattering component to the surfaces of Europa's leading hemisphere not seen in the 2PHG model solutions. However, for Europa's trailing hemisphere the 3P-HG model predicts that the surface is backscattering, while the $2 \mathrm{P}-\mathrm{HG}$ model predicts an additional forward scattering component. Both models show the same single scattering function variations with wavelength for Ganymede's trailing hemisphere, where the $0.47-\mu \mathrm{m}$ single scattering functions are nearly isotropic while the $0.55-\mu \mathrm{m}$ single scattering functions are highly backscattering. The only variations with model seen for Ganymede's leading hemisphere are those for the $0.55-\mu \mathrm{m}$ observations. Both models show that the single scattering function is backscattering at $0.47 \mu \mathrm{m}$, but the $2 \mathrm{P}-\mathrm{HG}$ model predicts that the $0.55-\mu \mathrm{m}$ single scattering function has a forward scattering component larger than that predicted by the $3 \mathrm{P}-\mathrm{HG}$ model. However, this may be due to the fact that there were no large phase angle observations at $0.55 \mu \mathrm{m}$ for the leading hemisphere of Ganymede. The 3P-HG model predicts that Callisto's trailing hemisphere is very strongly forward scattering while the $2 \mathrm{P}-\mathrm{HG}$ model shows some variations with wavelength, where the $0.47-\mu \mathrm{m}$ single scattering function has both forward and backscattering components and the $0.55-\mu \mathrm{m}$ single scattering function has a low broad backscattering lobe. The 3P-HG model predicts that Callisto's leading hemisphere is nearly isotropic, with a small narrow backscattering component, while the $2 \mathrm{P}-\mathrm{HG}$ model shows some variations with wavelength where the $0.47-\mu \mathrm{m}$ single scattering function is highly forward scattering and has a minor backscattering peak and the $0.55-\mu \mathrm{m}$ single scattering function has a very low, broad backscattering lobe. However, the phase curve data for Callisto's leading hemisphere have no large phase angle observations, so modeling results of this hemisphere need to be interpreted with caution. The 2P-HG model predictions for the single scattering function of Rhea are similar for both hemispheres at both wavelengths. It shows both a forward and backscattering component where the forward scattering component is the stronger of the two. The 3P-HG model predicts very different scattering functions between the two hemispheres. At $0.55 \mu \mathrm{m}$ the predicted single scattering function for the leading hemisphere has both components, as seen in the 2P-HG solutions; however, the forward scattering component is weaker than in the $2 \mathrm{P}-\mathrm{HG}$ solutions. For the trailing hemisphere at $0.55 \mu \mathrm{m}$ the $3 \mathrm{P}-\mathrm{HG}$ function predicts a completely backscattering scattering function. At $0.47 \mu \mathrm{m}$ the $3 \mathrm{P}-\mathrm{HG}$ function predicts an extremely large forward scattered component for Rhea's leading hemisphere, but a moderately forward scattering function for Rhea's trailing hemisphere, similar to that predicted for the $0.55-\mu \mathrm{m}$ leading hemisphere 3P-HG solution.

\section{DISCUSSION}

Hapke's model (Hapke 1981, 1984, 1986) is mathematically complex. The changes incurred in a phase curve derived with Hapke's model by changing the value of one model parameter can often be counterbalanced by changing a second parameter. This is why unique solutions to this model (especially for disk-integrated observations) require data sets that have a broad range of phase angle coverage. Large phase angle data (observations greater than $100^{\circ}$ solar phase) constrain the values of $\theta$-bar, $w$, and the single scattering function parameters. Moderate phase angle data (observations between $20^{\circ}$ and $100^{\circ}$ solar phase) combined with large phase angle observations also constrain the single particle scattering function, which in turns influences the values of $\theta$-bar and $w$. This is why the results of the satellite modeling (of those satellites with adequate larger phase angle coverage) that show substantial differences in the single scattering function between the 2P-HG Hapke model and the 3P-HG Hapke model also show differences in $w$ and/or $\theta$-bar between the two model results. In this section we focus our discussion on the single particle scattering results. However, a caveat must be stated. Not all 


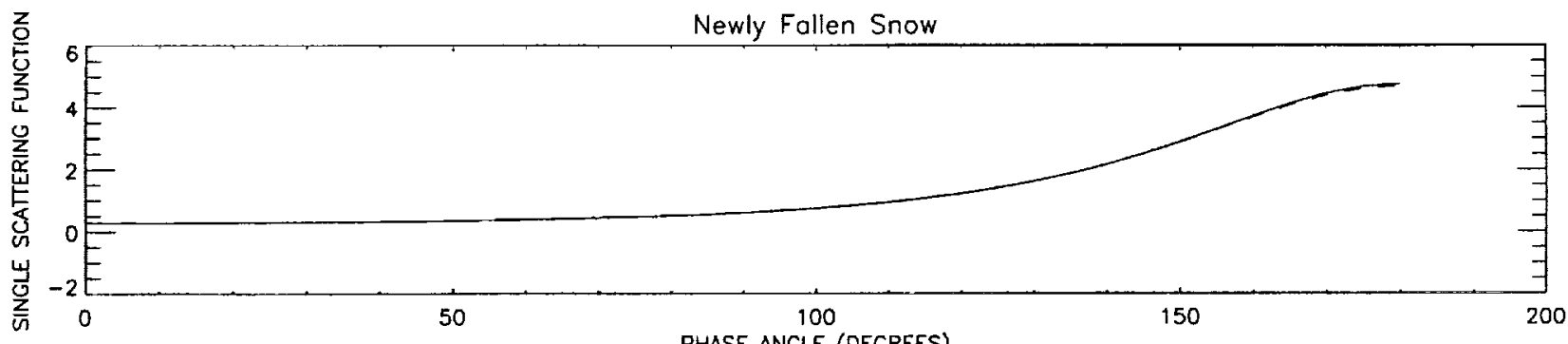

PHASE ANGLE (DEGREES)
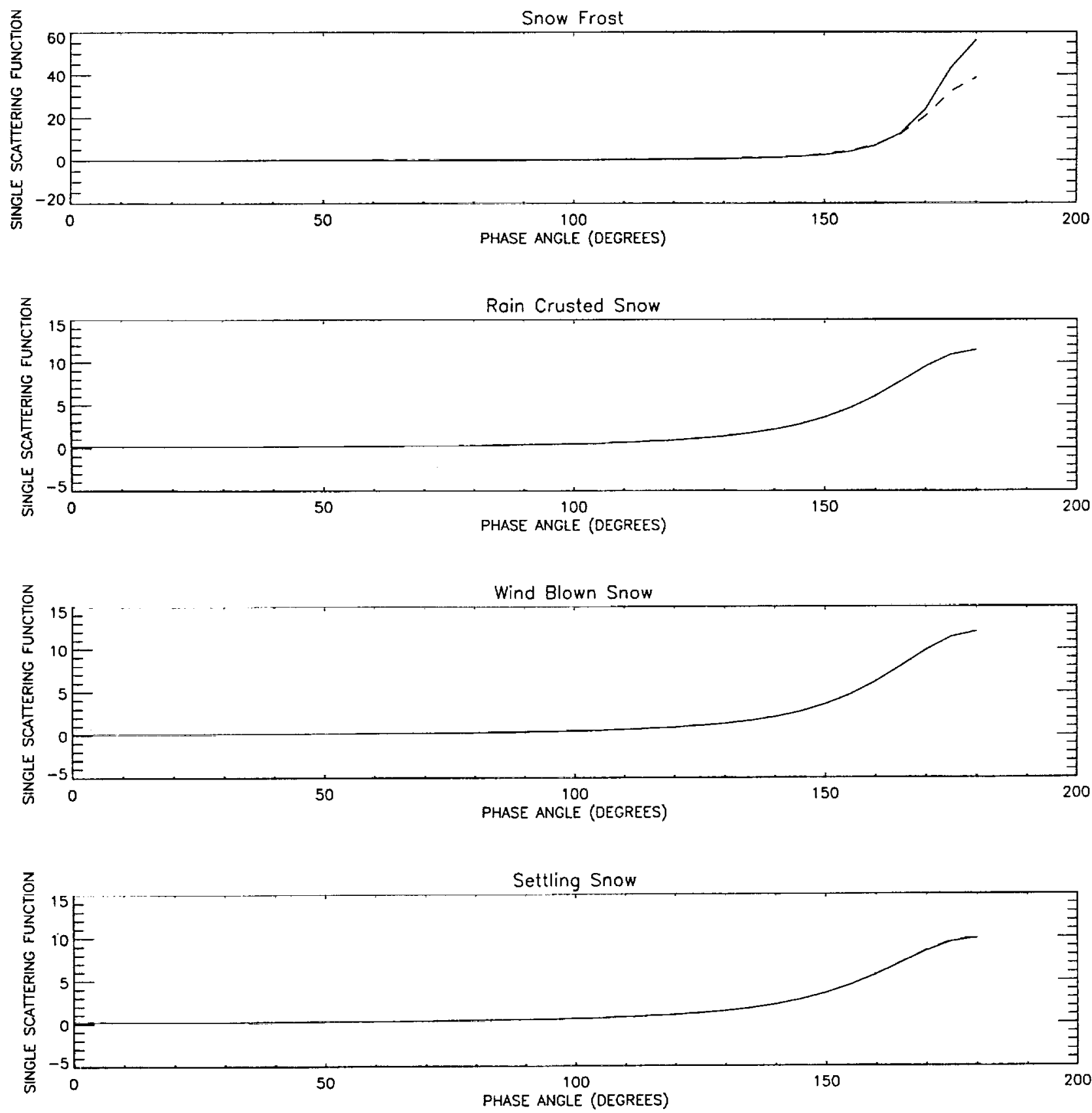

FIG. 8. The single particle scattering functions for the five terrestrial snow data sets where the solid line represents the $2 \mathrm{P}-\mathrm{HG}$ single scattering solution and the dashed line represents the 3P-HG single scattering solution. The graphs where no dashed line is seen indicates that the 1 wo single scattering functions are equivalent, and the dashed line is overlaid by the solid line. 

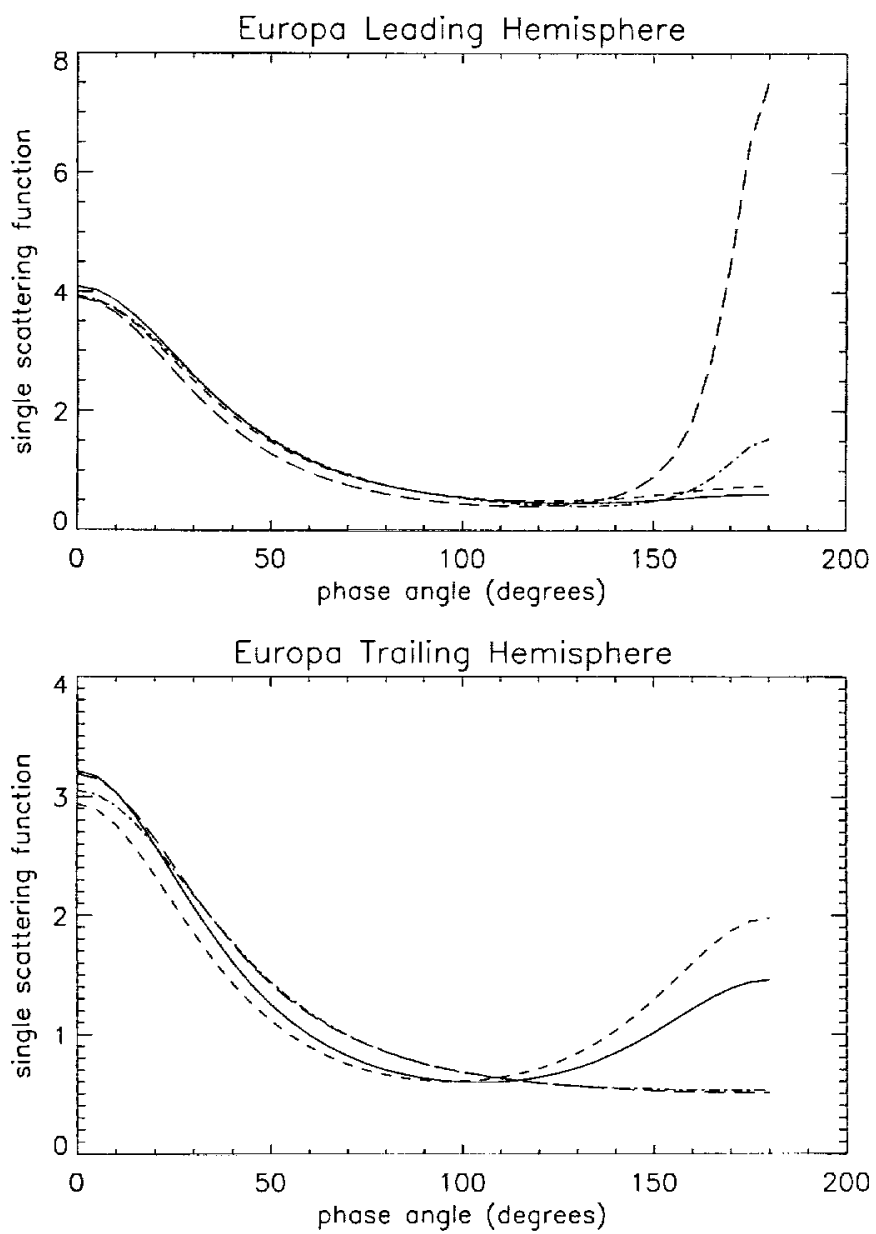

FIG. 9. The single particle scattering functions for Europa's leading and trailing hemispheres, plotted against the Hapke modeling solutions using the $2 \mathrm{P}-\mathrm{HG}$ single scattering function for the $0.47-\mu \mathrm{m}$ (solid line) and $0.55-\mu \mathrm{m}$ (short dashed line) observations and the Hapke modeling solutions using the 3P-HG single scattering function for the $0.47-\mu \mathrm{m}$ (long dashed line) and $0.55-\mu \mathrm{m}$ (dash-dot line) observations.

the satellite phase curves presented here have the necessary phase angle coverage to constrain adequately all the model parameters. Several are missing large phase angle observations, and therefore the values of the single scattering parameters along with the surface roughness parameter are poorly constrained, as demonstrated by the error bars listed in Table V. Domingue and Verbiscer (1997) discuss this in greater detail. We have chosen to present these phase curves in order to demonstrate the effects of poor phase angle coverage on the modeling results. The following discussion is based on the model parameter values given in Table II; however, the error bars in Table V provide a measure of the accuracy of our interpretations based on the available phase angle coverage.

One of the basic assumptions in Hapke's model regarding the scattering behavior of planetary regoliths is that while the singly scattered component may be anisotropic, the multiply scattered component can be approximated to first order as isotropic. As the albedo of a surface increases, the role of multiple scattering becomes more important; therefore, the assumption of isotropic multiple scattering for high albedo surfaces, such as snow and many icy satellites, is no longer valid (Mishchenko 1994, Goguen 1997, Verbiscer and Helfenstein 1997). The model used in this study, the results presented in Tables II-V, and the text assume that the multiply scattered component is isotropic. If the anisotropy in the multiply scattered component is not coupled to the anisotropy in the single scattered component, then correcting for anisotropic scattering in the multiply scattered component introduces a second scattering function and thereby one to three additional parameters into Hapke's model. The icy satellite data are not extensive enough to constrain the number of parameters of such a model. However, if the assumption is made that the anisotropy in the single and multiply scattered components can be described by the same scattering function, then there are no additional parameters (Verbiscer 1991).
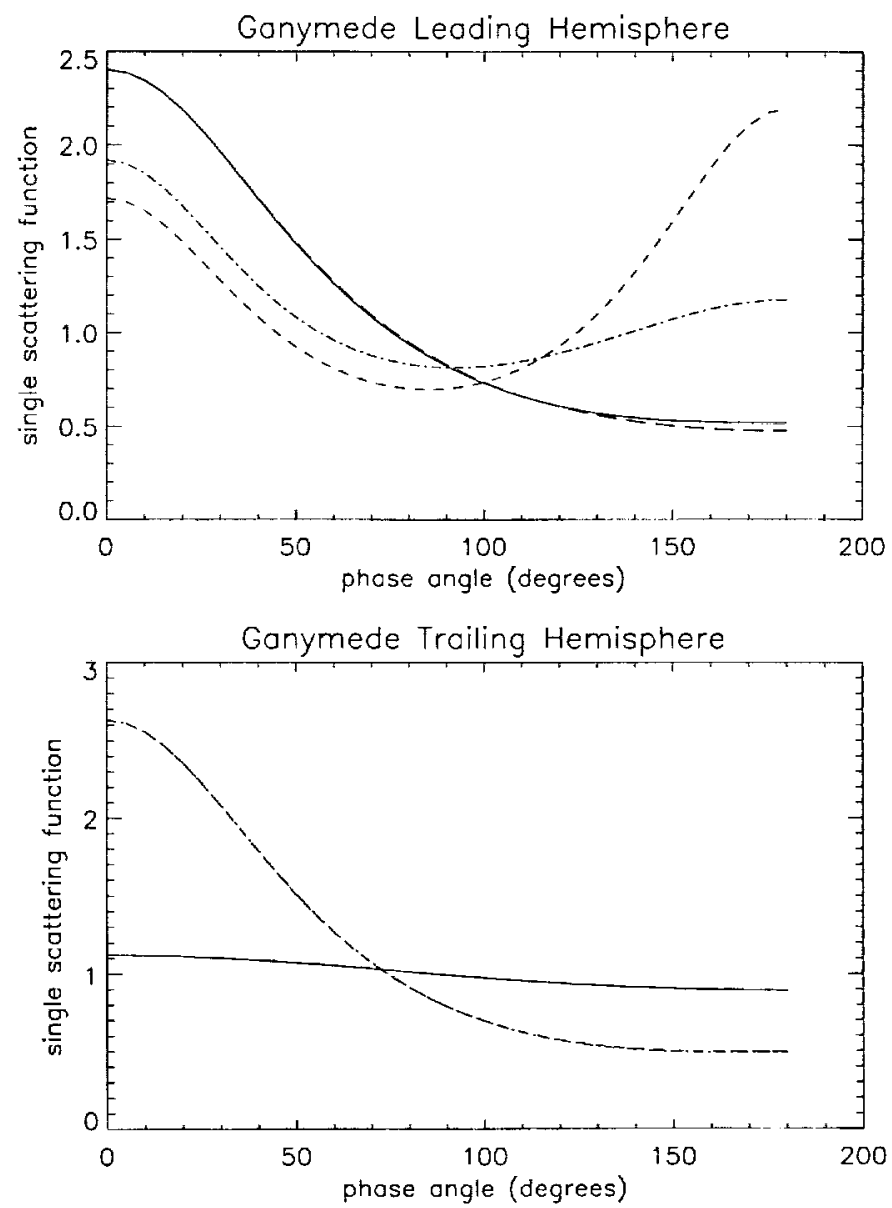

FIG. 10. Same as described in the legend for Fig. 9, for Ganymede. 

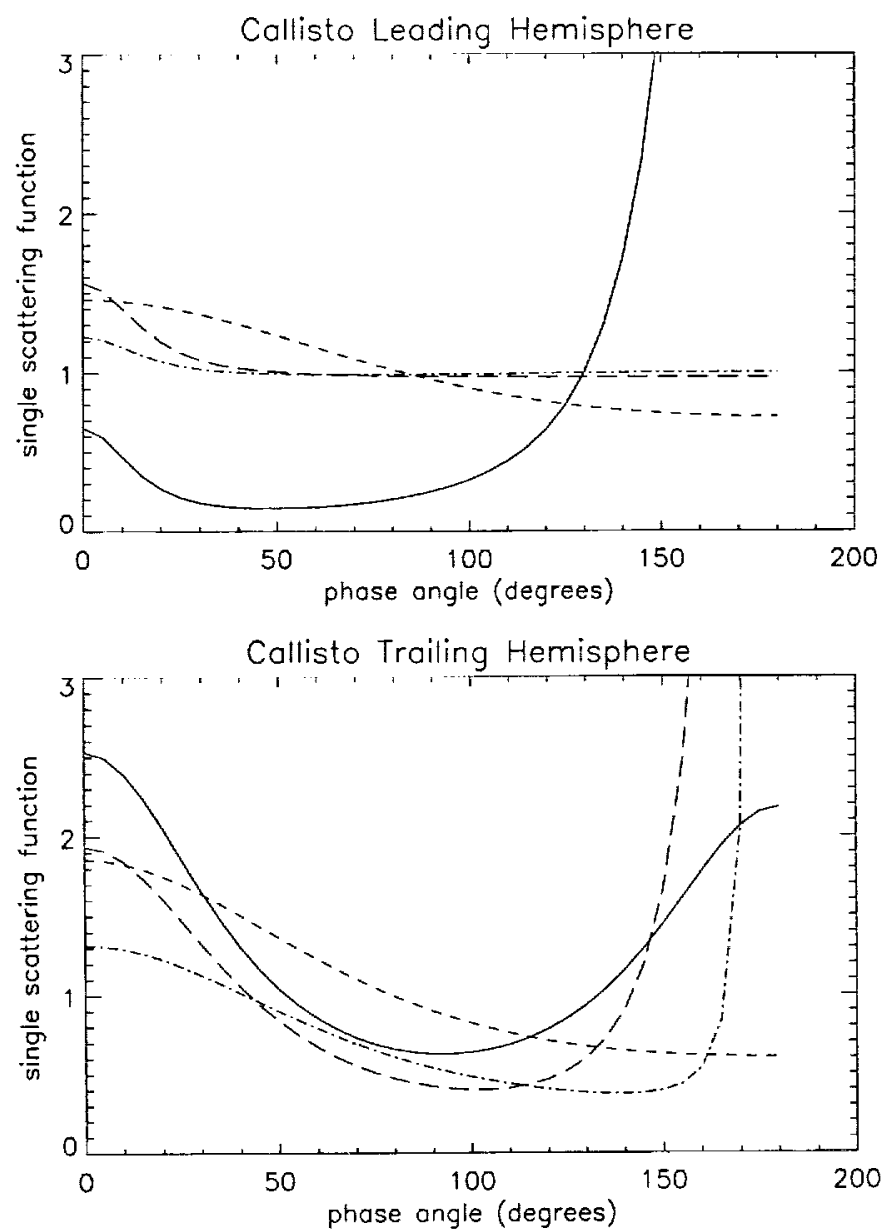

FIG. 11. Same as described in the legend for Fig. 9, for Callisto.

We refit the snow data using a single-parameter HenyeyGreenstein function to describe the anisotropic scattering of both the singly and the multiply scattered components. Since our initial results of modeling the snow data showed that both the 2P-HG and the 3P-HG functions gave solutions which reduced to a single parameter HenyeyGreenstein function we felt that such comparisons would be valid. In addition we set bo $=0$ since we found that in the isotropic multiple scattering case the solutions with bo $=0$ and bo $\neq 0$ showed no substantial differences. When we compared our anisotropic multiple scattering results to our isotropic multiple scattering results we see no difference outside of our error bars for the scattering function or the single scattering albedo; however, the rms values for the anisotropic case are nearly twice those for the isotropic case. Since the anisotropic multiple scattering model gives similar results (well within the error bars but with higher rms values) to the isotropic model for bright terrestrial snows, we hypothesized that the anisotropic model would produce similar results as the isotropic model for the darker icy satellite surfaces.
Domingue and Verbiscer (1997) applied both an isotropic and anisotropic multiple scattering Hapke model to the phase curve observations of Europa and Ganymede, the brightest of the Galilean satellites. Since Rhea is comparable in albedo to Europa, we also applied an anisotropic model (using a 3P-HG scattering function, as was used in the Domingue and Verbiscer analysis) to the Rhea leading hemisphere phase curves. No application was made to Rhea's trailing hemisphere observations since the large phase angle coverage is less than $50^{\circ}$. The anisotropic multiple scattering modeling results for Rhea are given in Table VI and compared to the isotropic multiple scattering results. The isotropic versus anisotropic multiple scattering modeling results of the icy satellites are commensurate with the corresponding modeling results for the terrestrial snows. The most substantial differences (differences outside the error bars) between the isotropic and the anisotropic results for the icy satellites were for phase angle ranges not constrained by the data, such as the forward scattering direction $\left(\alpha>120^{\circ}\right)$. Comparisons of the pre-
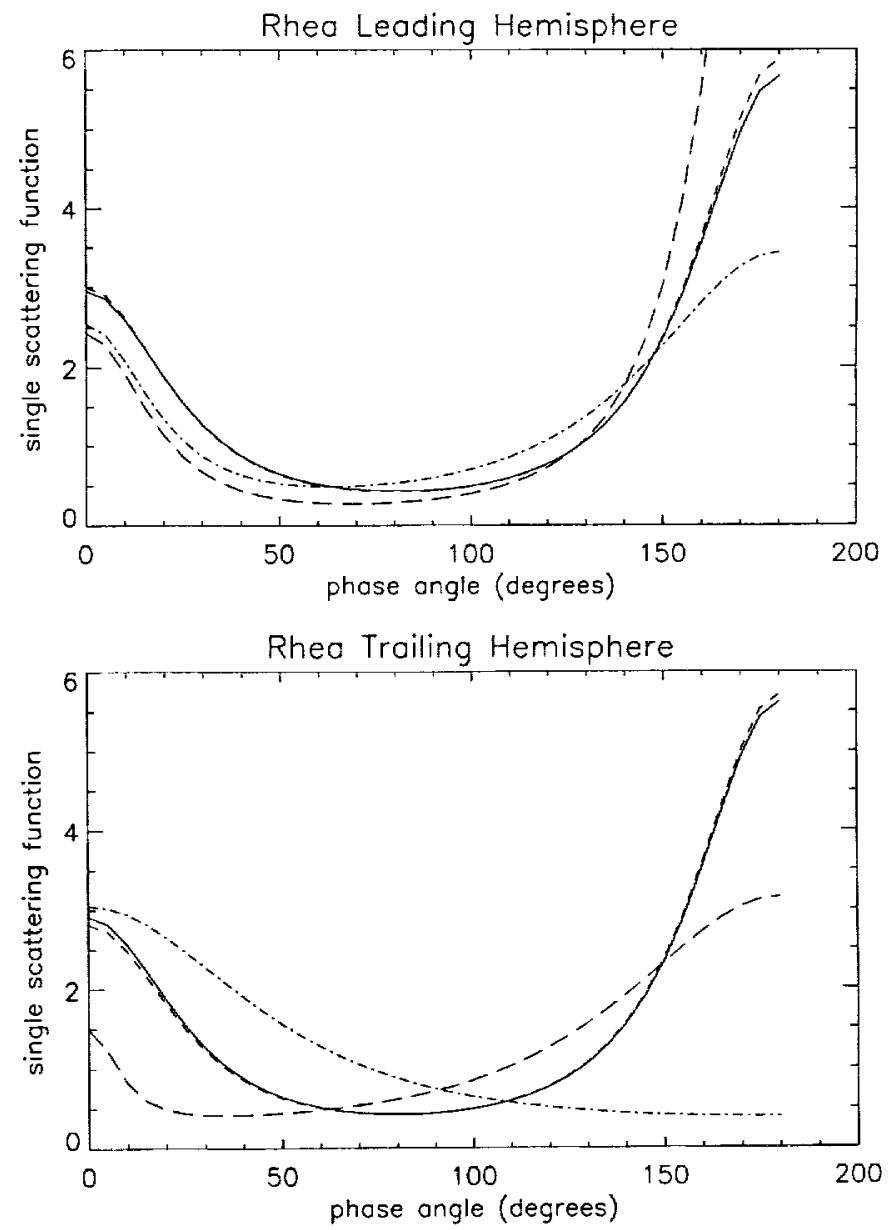

FIG. 12. Same as described in the legend for Fig. 9, for Rhea. 


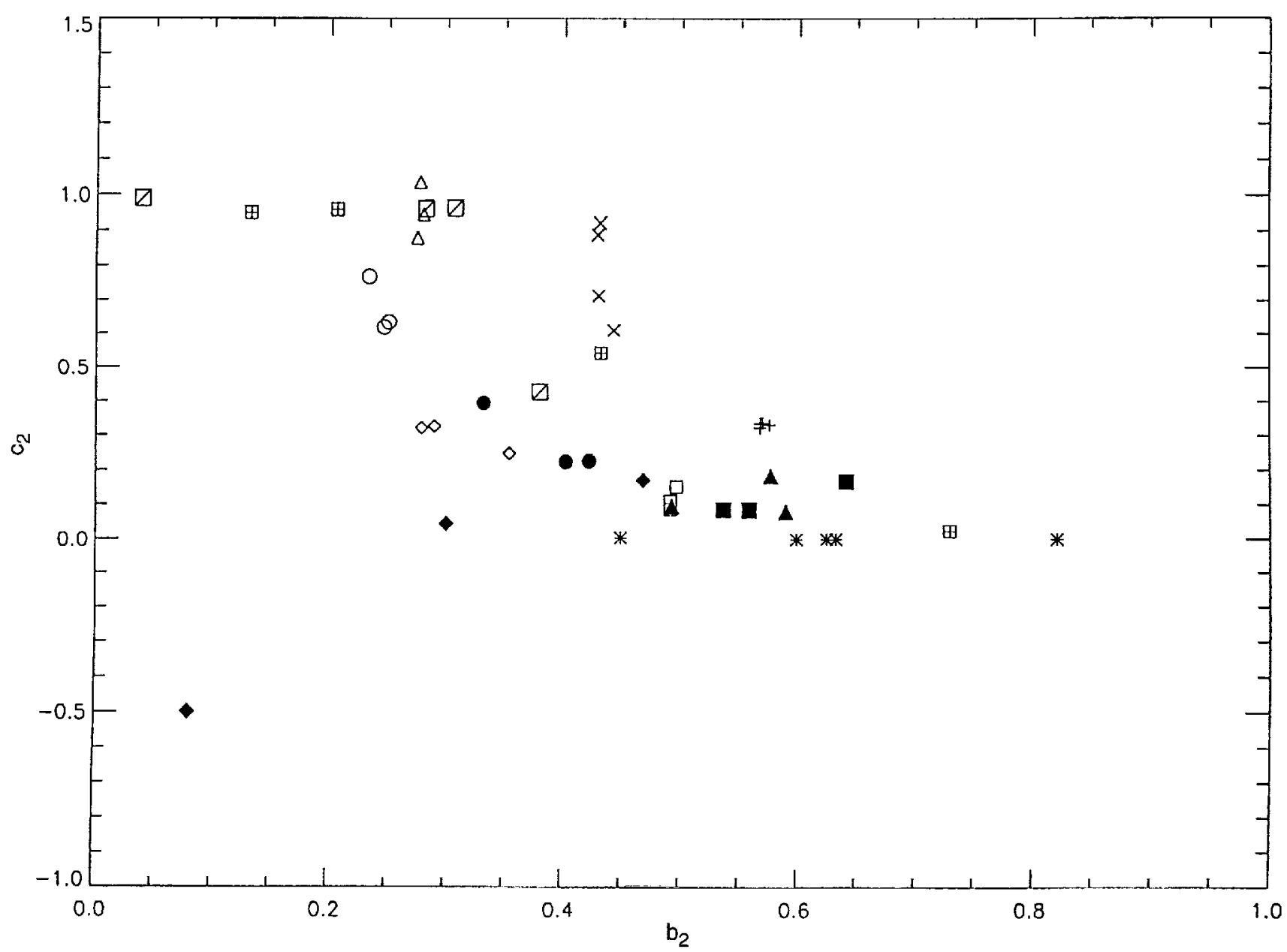

FIG. 13. A plot of the two-dimensional 2P-HG single scattering parameter space $\left(b_{2}\right.$ versus $\left.c_{2}\right)$ of Hartman and Domingue (1995) solutions to the McGuire and Hapke (1995) artificial particles ( $\boldsymbol{\boldsymbol { \omega }}$, smooth, clear sphere; $\boldsymbol{\bullet}$, rough, clear sphere; $\boldsymbol{\Delta}$, pitted, clear sphere; $\bullet$, irregular shaped particle; $\square$, sphere with low density of internal scatterers; $O$, sphere with moderate density of internal scatterers; $\Delta$, sphere with high density of internal scatterers; $\diamond$, agglutinate) versus our $2 \mathrm{P}-\mathrm{HG}$ single scattering parameter results for terrestrial snow (*); Europa $(\mathrm{X})$; Ganymede $(\square)$; Callisto $(\mathbb{\theta})$, and Rhea $(+)$.

dicted phase curves and of the single scattering function were nearly identical between the isotropic and anisotropic model parameters within those phase angles covered by the data sets. Any divergences in the predicted phase curve behavior or the single scattering function behavior occur only at phase angle ranges beyond those constrained by observations. There were also significant differences (differences outside of the error bars) in the opposition amplitude parameter (bo) for both Europa and Ganymede (Domingue and Verbiscer 1997). The differences between the isotropic bo values and the anisotropic bo values show no systematic trend; however, the isotropic values show smaller differences in bo between the two wavelength solutions than between the anisotropic model solutions. The parameter values between the isotropic and anisotropic multiple scattering models for the icy satellites are qualitatively similar; they predict similar single scattering functions for phase angle ranges within the observations, similar particle opacities (bo $<1$ is transparent, bo $\geq 1$ is opaque), similar surface roughness, and similar single scattering albedos. The quantitative differences, such as predicted single scattering behavior beyond the phase angle coverage and the degree of opacity, can be attributed to the different mathematical coupling of the scattering function to the opposition parameters between the isotropic and the anisotropic models. Predictions of scattering behavior for phase angles not contained in a data set are going to be strongly model dependent. For the analyses presented in this study we have chosen the isotropic multiple scattering model since the solutions are qualitatively 


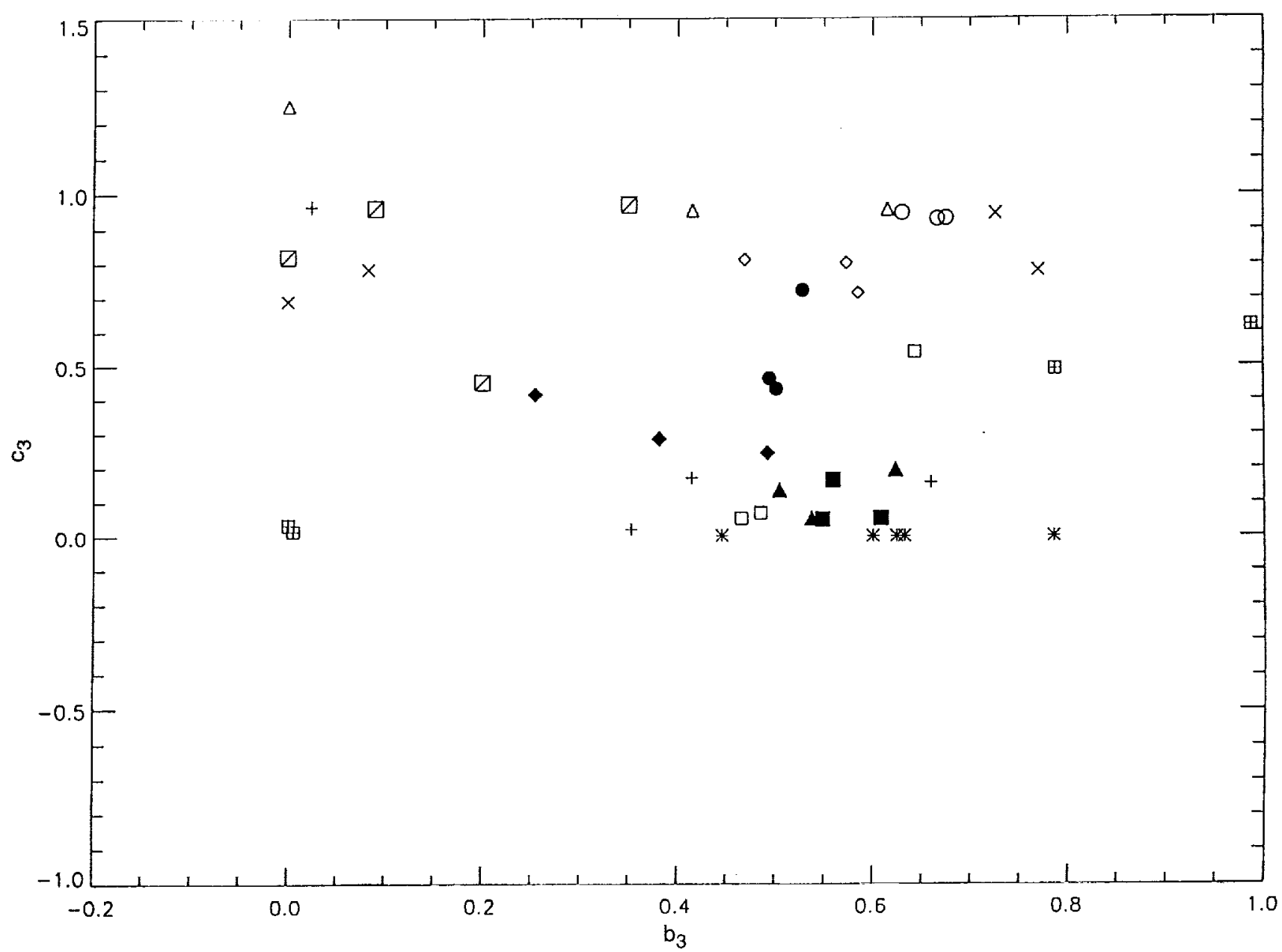

FIG. 14. Same as described in the legend for Fig. 13, for the three-dimensional 3P-HG single scattering parameter space defined by $b_{3}$ and $c_{3}$.

similar to the anisotropic results and, in addition, the isotropic solutions have opposition width parameter $(h)$ values and macroscopic roughness parameter $(\theta$-bar) values which are invariant with wavelength, consistent with their physical definitions.

Hartman and Domingue (1997) have shown that there are no differences in the quality of fit between the $2 \mathrm{P}-\mathrm{HG}$ and the $3 \mathrm{P}-\mathrm{HG}$ functions when applied to the McGuire and Hapke (1995) single scattering laboratory data. Our results in modeling the terrestrial snows support their findings. McGuire and Hapke (1995) and Hartman and Domingue (1997) plotted the single scattering parameter values against each other to see if specific particle types cluster in certain areas of single scattering parameter space. The purpose of this was to obtain a mechanism for correlating the single scattering parameters with physical particle structures and textures. Figure 13 is a plot of the $2 \mathrm{P}-\mathrm{HG}$ parameter space that includes the results of Hartman and Domingue (1997) with our results for the terrestrial snows and the satellites. Figures 14 through 16 are similar plots of the 3P-HG parameter space.

Figure 13 shows that our $2 \mathrm{P}-\mathrm{HG}$ model scattering results suggest that terrestrial snows are most similar in structure to the Hartman and Domingue (1997) category A type particles. Category A type particles are those which depart the least from a smooth, clear sphere. The frost values, however, plot outside of the laboratory defined areas. Frost is shown to have higher values of $b_{2}$ than any of the Hartman and Domingue particle categories; however, it does have similar $c_{2}$ values to their category $A$ particles. The 2P-HG values for the icy satellites plot outside of the laboratory defined parameter space. This indicates that there are a variety of particle physical types present on their surfaces and the single scattering parameters represent an average. The $2 \mathrm{P}-\mathrm{HG}$ values for Europa indicate that the structural characteristics of its regolith particles are a combination of irregular shape and presence of internal scatterers. These values also indicate a higher presence of internal 


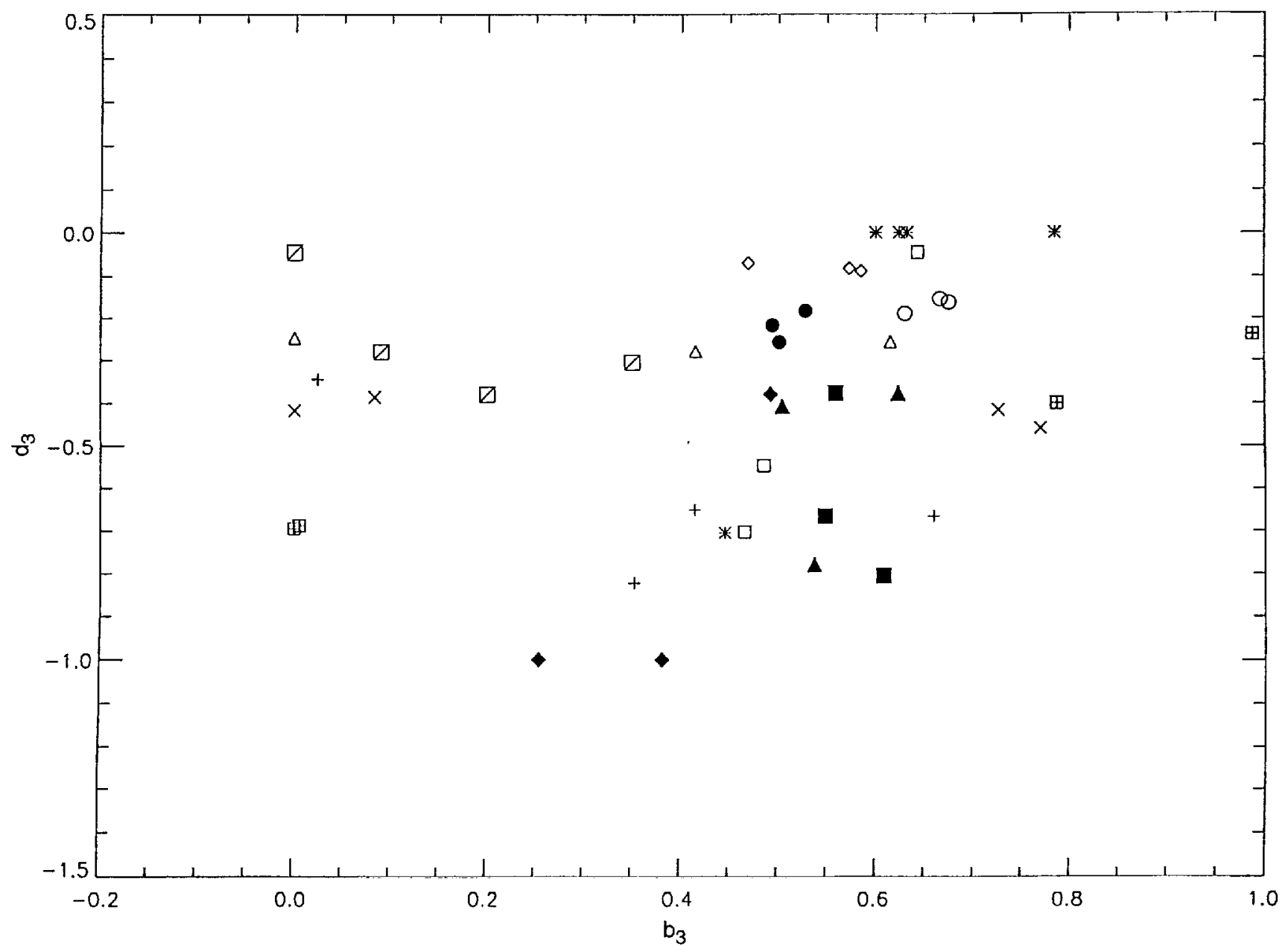

FIG. 15. Same as described in the legend for Fig. 13, for the three-dimensionaI 3P-HG single scattering parameter space defined by $b_{3}$ and $d_{3}$.

scatterers for the leading hemisphere with respect to the trailing hemisphere. This is consistent with the preferential bombardment of the trailing hemisphere by magnetospheric ions (Sack et al. 1991, 1992). The 2P-HG results for Ganymede and Callisto are scattered and predict different particle types dependent on wavelength. The particle types predicted should be independent of wavelength. However, there was no large phase angle coverage for Ganymede in the $0.55-\mu \mathrm{m}$ observations and none for Callisto's leading hemisphere, which may account for some of these inconsistencies. The Rhea parameters most closely correlate to the category A type particles, with possible departures in shape from perfect spheres.

Figures 14 through 16 plot our 3P-HG modeling results against the 3P-HG laboratory modeling results of Hartman and Domingue (1997). These figures clearly demonstrate that the structure of terrestrial snow and the regolith particles of the satellites we have chosen to study have nothing in common with the structures of the laboratory particles of McGuire and Hapke (1995). Our snow results define a new area in the parameter space. The parameter values for the icy satellites do not fall in this region, supporting the Verbiscer and Veverka (1990) hypothesis that the grain structures and textures of outer Solar System water ices are very different from terrestrial analogs and the laboratory particle structures studied by McGuire and Hapke (1995). The only laboratory particle which falls into the icy satellite region is that of the high albedo $(w=0.67)$ sphere with a high density of internal scatterers. This is a strong indication that the particle structures of icy satellite regoliths are not uniform, but consist of a variety of particle structure and texture types.

Helfenstein et al. (1997) analyzed the McGuire and Hapke laboratory data using a 3P-HG function in an effort to find an albedo dependence within the particle scattering phase functions. In their study Helfenstein et al. (1997) 


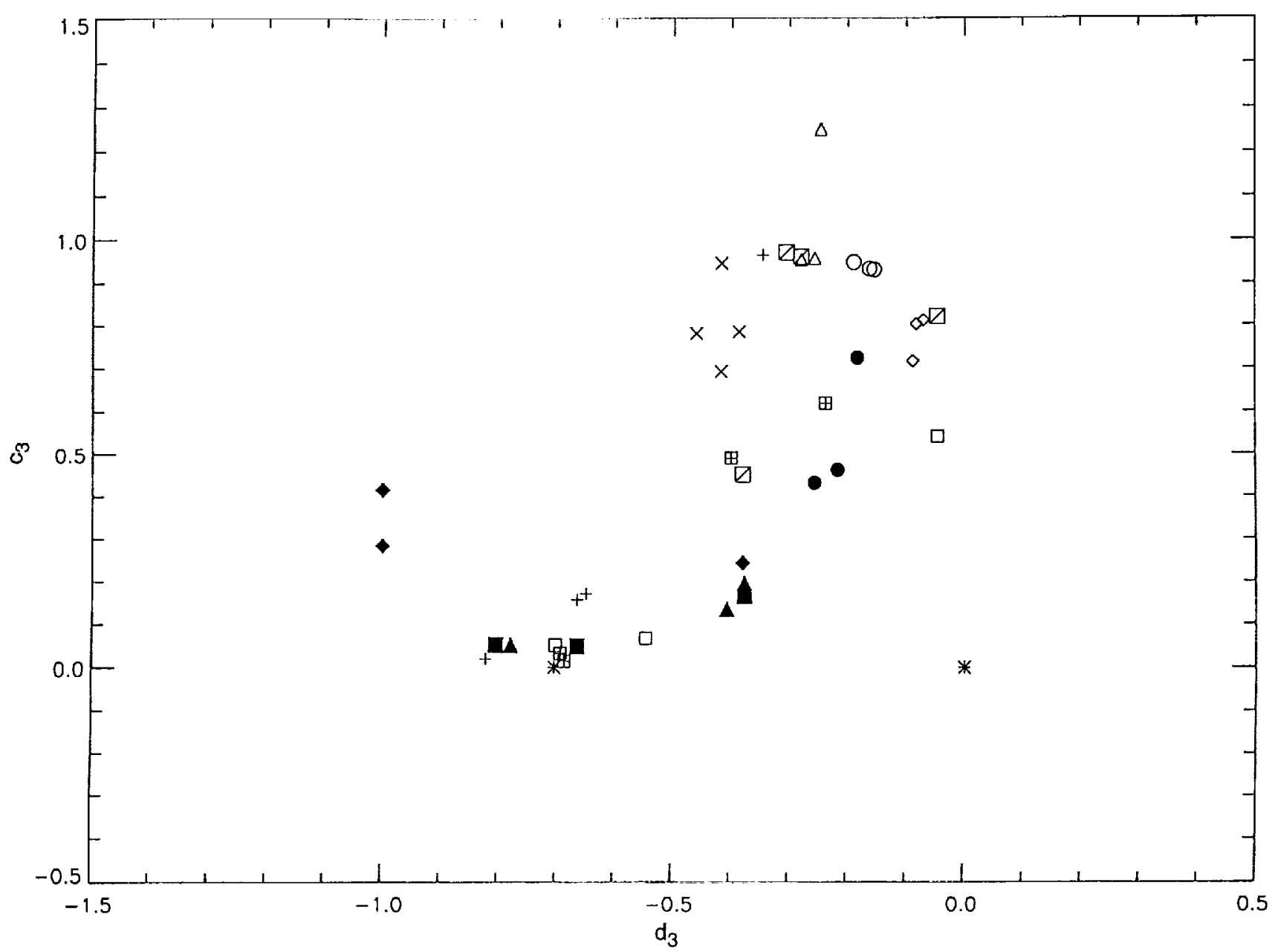

FIG. 16. Same as described in the legend for Fig. 13, for the three-dimensional 3P-HG single scattering parameter space defined by $d_{3}$ and $c_{3}$.

define an effective asymmetry parameter, $g_{\mathrm{eff} 3}$, such that

$g_{\mathrm{crf3}}=\langle\cos \theta\rangle=\frac{\int_{0}^{\pi} P_{3}(\theta) \cos \theta \sin \theta \delta \theta}{\int_{0}^{\pi} P_{3}(\theta) \sin \theta \delta \theta}=\left(1-c_{3}\right) b_{3}+c_{3} d_{3}$,

where $\theta$ is the scattering angle and is related to the phase angle by $\theta=\pi-\alpha$. For the two parameter $\mathrm{HG}$ function $d_{3}=-b_{3}$, thus $g_{\mathrm{eff} 2}=b_{2}\left(1-2 c_{2}\right)$. Figure 17 plots $g_{\mathrm{efr} 3}$ for the Hartman and Domingue (1997) laboratory results along with our results for the terrestrial snow data and the satellite observations. These plots demonstrate that the particle structures for terrestrial snow and planetary regoliths are much more complicated than the structures of the McGuire and Hapke (1995) laboratory particles and that the structures of the icy regoliths measured in this study are very different than the structure of terrestrial snow and frost.

\section{CONCLUSIONS}

Our comparisons of the single particle scattering behavior of terrestrial snows and icy satellite regoliths to the laboratory particle scattering measurements of McGuire and Hapke (1995) demonstrate that the differences between icy satellite regoliths and their terrestrial counterparts are due to particle structures and textures. Terrestrial snow particle structures define a region in the single particle scattering function parameter space separate from the regions defined by the McGuire and Hapke (1995) artificial laboratory particles. The particle structures and textures of the grains composing icy satellites regoliths are not simple or uniform but consist of a variety of particle structure and texture types, some of which may be a combination of the particle types investigated by McGuire and Hapke (1995). 
Buratti, B. 1991. Ganymede and Callisto: Surface textural dichotomies and photometric analysis. Icarus 92, 312-323.

Buratti, B., and J. Veverka 1983. Voyager photometry of Europa. Icarus 55, 93-110.

Buratti, B., and J. Veverka 1984. Voyager photometry of Rhea, Dione, Tethys, Enceladus, and Mimas. Icarus 58, 254-264.

Buratti, B., R. M. Nelson, and A. L. Lane 1988. Surficial textures of the Galilean satellites. Nature (London) 33, 148-151.

Buratti, B. J., F. Wong, and J. A. Mosher 1990. Surface properties and photometry of the uranian satellites. Icarus 84, 203-214.

Calvin, W. M., R. N. Clark, R. H. Brown, and J. R. Spencer 1995. Spectra of the icy Galilean satellites from 0.2 to $5 \mu \mathrm{m}$ : A compilation, new observations, and a recent summary. J. Geophys. Res. 100, 19041-19048.

Clark, R. 1980. Ganymede, Europa, Callisto, and Saturn's rings: Compositional analysis for reflectance spectroscopy. Icanis 44, 388-409.

Domingue, Deborah, and Anne Verbiscer 1997. Re-analysis of the solar phase curves of the icy Galilean satellites. Icarus 128, 49-74.

Domingue, D. L., B. W. Hapke, G. W. Lockwood, and D. T. Thompson 1991. Europa's phase curve: Implications for surface structure. Icarus 90, 30-42.

Domingue, D. L., G. W. Lockwood, and D. T. Thompson 1995. Surface textural properties of icy satellites: A comparison between Europa and Rhea. Icarus 115, 228-249.

Goguen, J. D. 1997. A quantitative test of the applicability of independent scattering to high albedo planetary regoliths. Icarus, submitted.

Hapke, B. 1981. Bidirectional reflectance spectroscopy. 1. Theory. $J$. Geophys. Res. 86, 3039-3054.

Hapke, B. 1984. Bidirectional reflectance spectroscopy. 3. Correction for macroscopic roughness. Icarus 59, 41-59.

Hapke, B. 1986. Bidirectional reflectance spectroscopy. 4. The extinction coefficient and the opposition effect. Icarus 67, 264-280.

Hartman, B., and D. Domingue 1997. Scattering of light by individual particles and the implications for models of planetary surfaces. Icaris, submitted.

Helfenstein, P. 1986. Derivation and Analysis of Geological Constraints on the Emplacement and Evolution of Terrains on Ganymede from Applied Differential Photometry. Thesis, Brown Univ., Providence, RI.
Helfenstein, P., J. Hillier, and J. Veverka 1997. Albedo dependence of particle phase functions for planetary regoliths. Icarus, submitted.

Helfenstein, P., J. Veverka, and P. Thomas 1988. Uranus satellites: Hapke parameters from Voyager disk-integrated photometry. Icarus 74, 231-239.

Hillier, J., P. Helfenstein, A. Verbiscer, and J. Veverka 1991. Voyager photometry of Triton: Haze and surface photometric properties. $J$. Geophys. Res. 96, 19203-19209.

Hobbs, P. 1974. Ice Physics. Clarendon, Oxford.

Middleton, W. E. K., and A. G. Mungall 1952. The luminous directional reflectance of snow. J. Opt. Soc. Am. 42, 572-579.

Mishchenko, M. I. 1994. Asymmetry parameters of the phase function for densely packed scattering grains. J. Quant. Spectrosc. Radiat. Transfer 52(1), 95-110.

McGuire, A. F., and B. W. Hapke 1995: An experimental study of light scattering by large, irregular particles. Icarus 113, 134-155.

Sack, N. J., J. W. Boring, R. E. Johnson, R. A. Baragiola, and M. Shi 1991. Alteration of the UV-visible reflectance spectra of $\mathrm{H}_{2} \mathrm{O}$ ice by ion bombardment. J. Geophys. Res. Planets E96(2), 17535-17539.

Sack, N. J., R. E. Johnson, J. W. Boring, and R. A. Baragiola 1992. The effect of magnetospheric ion bombardment on the reflectance of Europa's surface. Icarus 100, 534-540.

Verbiscer, A. 1991. Photometry of Icy Satellite Surfaces. Ph.D. thesis, Cornell Univ., Ithaca, NY.

Verbiscer, A., and P. Helfenstein 1997. Reflectance spectroscopy of icy surfaces. In Ices in the Solar System (B. Schmitt, C. deBergh, and M. Festou, Eds.). Kluwer Academic, Dordrecht, in press.

Verbiscer, A. J., and J. Veverka 1989. Albedo dichotomy of Rhea: Hapke analysis of Voyager photometry. Icarus 82, 336-353.

Verbiscer, A. J., and J. Veverka 1990. Scattering properties of natural snow and frost: Comparison with icy satellite photometry. Icarus 88, $418-428$.

Verbiscer, A., P. Helfenstein, and J. Veverka 1990. Backscattering from frost on icy satellites in the outer solar system. Nature (London) 347, 162-164.

Veverka, J., P. Thomas, P. Helfenstein, R. H. Brown, and T. V. Johnson 1987. Satellites of Uranus: Disk integrated photometry from Voyager imaging observations. J. Geophys. Res. 92, 14895-14904. 

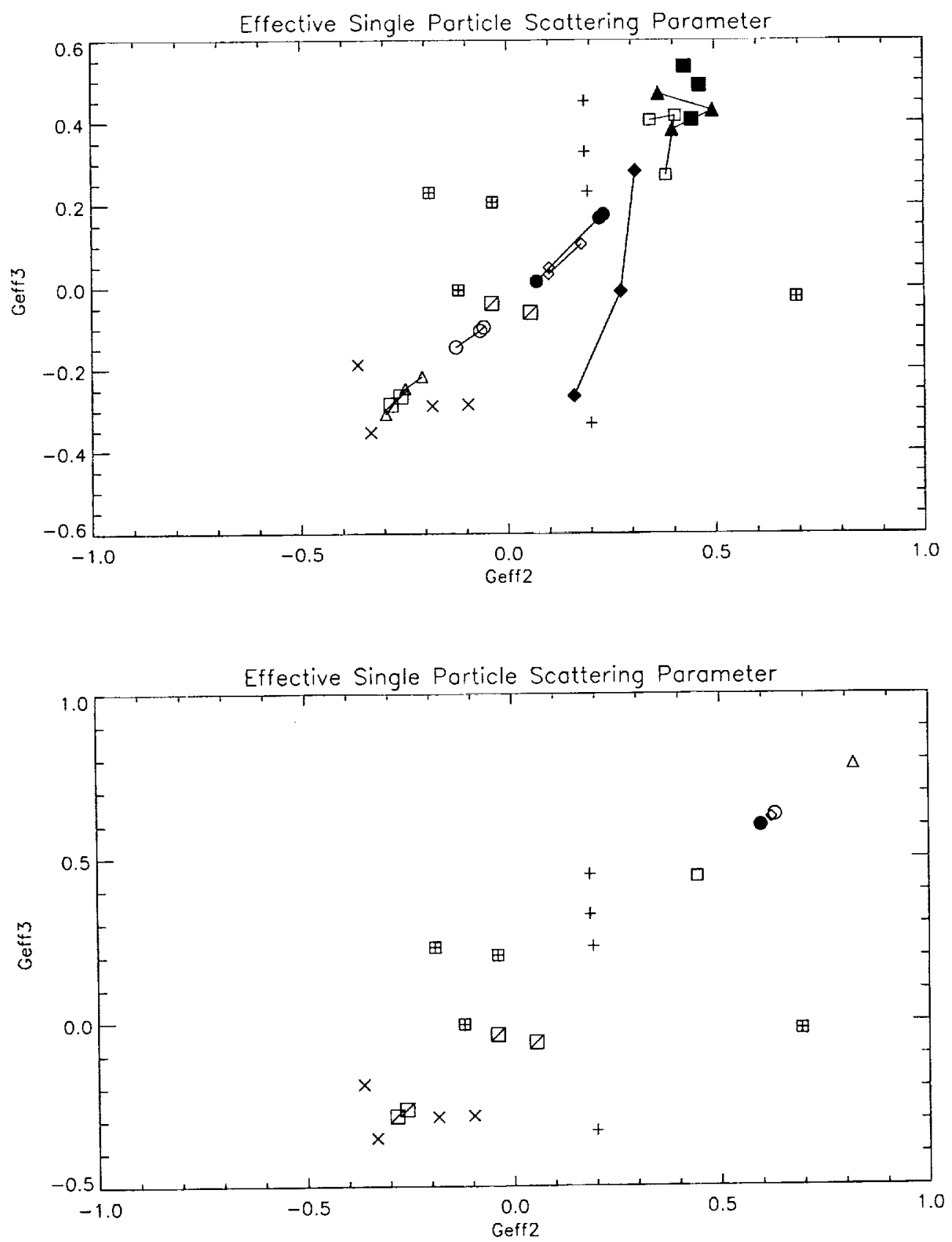

FIG. 17. Plots of the 2P-HG effective scattering parameter $\left(g_{\text {eff }}\right)$ versus the $3 \mathrm{P}-\mathrm{HG}$ effective scattering parameter $\left(g_{\text {eff }}\right)$ for: (top) Hartman and Domingue (1995) solutions to the McGuire and Hapke (1995) artificial particles (same symbols as described in the legend to Fig. 13 and

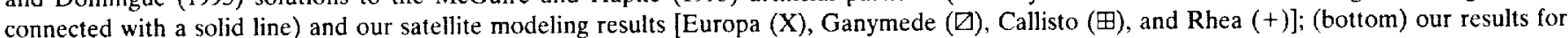
terrestrial snow $(\square$, newly fallen snow; $\Delta$, snow frost; $\diamond$, rain crusted snow; $O$, wind blown snow; $\bullet$, settling snow) versus our satellite modeling results (symbols same as described for top graph).

\section{ACKNOWLEDGMENTS}

The authors thank John Hillier and an anonymous referee for insightful reviews. The work of B. Hartman was accomplished under the LPI Undergraduate Summer Internship Program. The work of A. Verbiscer was accomplished under NASA Grant NASA NAGW-3591. LPI Contribution 919.

\section{REFERENCES}

Buratti, B. 1984. Voyager disk resolved photometry of the saturnian satellites. Icarus 59, 392-405.

Buratti, B. 1985. Application of a radiative transfer model to bright icy satellites. Icarus 61, 208-217. 